\title{
Adaptive Speed Control Design for Brushed Permanent Magnet DC Motor Based on Worst-Case Analysis Approach
}

\author{
Sheng Zeng \\ Research and Development Department of Critical Care Engineering, CareFusion, Yorba Linda, CA 92886, USA \\ Correspondence should be addressed to Sheng Zeng; sheng.zeng@carefusion.com
}

Received 30 April 2012; Revised 23 July 2012; Accepted 23 August 2012

Academic Editor: Pedro Ribeiro

Copyright (C) 2013 Sheng Zeng. This is an open access article distributed under the Creative Commons Attribution License, which permits unrestricted use, distribution, and reproduction in any medium, provided the original work is properly cited.

\begin{abstract}
This paper presents the adaptive controller design for brushed permanent magnet DC motor used in velocity-tracking applications based on worst-case approach. We first formulate the robust adaptive control problem as a nonlinear $H^{\infty}$-control problem under imperfect state measurement, and then solve it using game-theoretic approach. The controller guarantees the boundedness of closed-loop signals with bounded exogenous disturbances, and achieves desired disturbance attenuation level with respect to the unmeasured exogenous disturbance inputs and the measured disturbance inputs. The strong robustness properties are illustrated by a simulation example.
\end{abstract}

\section{Introduction}

Permanent magnet brushed DC (PMBDC) motors are widely used in real world applications, and particularly in the highvolume commercial products, which is due to the PMBDC motors' better cost-to-performance ratio than most other motors. However, the structure of these permanent magnetic motors induces several uncertainties such as unmodelled nonlinear dynamics and undesired commutation/dent torques. The magnets lose/degrade their magnetic properties over time, and the motor constant varies with the changes of temperature and operating conditions. Moreover, the ever increasing control demands for high-efficiency and low-cost required to run the PMBDC motor at its technical limit. All above design challenges call for a robust adaptive controller for motion control applications over a wide range of operating conditions.

Adaptive control attracted a lot of research attention in control theory since 1970s. The classic adaptive control design based on the certainty equivalence approach leads to structurally simple adaptive controllers $[1,2]$, and its effectiveness for linear systems with or without stochastic disturbance inputs has been demonstrated when long-term asymptotic performance is considered [3]. However, early designs based on this approach were not robust to exogenous disturbance inputs and unmodeled dynamics [4]. Then, the stability and the performance of the closed-loop system becomes an important issue, which motivated the study of robust adaptive control in the 1980s and 1990s.

Robust adaptive control design is one of the most important research topics in control theory, which addresses the design of controllers that are robust to model uncertainties, and insensitive to the exogenous disturbances. Various adaptive controllers were modified to render the closed-loop systems robust [5-10]. Despite their successes, they however fell short of addressing the disturbance attenuation property of the closed-loop system. Worst-case analysis-based robust adaptive-control design was motivated by the success of the game-theoretic approach to $H^{\infty}$-optimal control problems [11] in late 1990s, which addresses the disturbance attenuation property directly. In this approach, the robust adaptive control problem is formulated as a nonlinear $H^{\infty}$-control problem under imperfect state measurements. By cost-to-come function analysis, it is converted into an $H^{\infty}$-control problem with full information measurements. This full information measurements problem is then solved using nonlinear design tools for a suboptimal solution. This design paradigm has been applied to worst-case parameter identification problems [12], which has led to new classes of parametrized identifiers for linear and nonlinear systems [13-16]. It has also been 
applied to adaptive control problems [17-21], and offered a promising tool to system subjected to uncertainties.

In this paper, we study the adaptive control design for permanent magnet DC motor based on worst-case analysis approach. We first model the permanent magnet DC motor servo system which is linear in all of the uncertainties. We then formulate the robust adaptive control problem as a nonlinear $H^{\infty}$-control problem under imperfect state measurements, and apply the cost-to-come function analysis to derive the worst-case identifier and state estimator. The control design of the plant subsystem follows [22], and the adaptive controller can be obtained by the integrator backstepping methodology. The robust adaptive controller achieves asymptotic tracking if the disturbances are bounded and of finite energy, and guarantees the stability of the closedloop system with respect to the bounded disturbance inputs and the initial conditions. Furthermore, the closeld-loop system admits a guaranteed disturbance attenuation level with respect to the exogenous disturbance inputs and the measured disturbances, where ultimate lower bound for the achievable attenuation performance level is only related to the noise intensity in the measurement channel of the plant system.

The balance of the paper is organized as follows. In Section 2, we present the formulation of the adaptive control problem and discuss the general solution methodology, then we obtain parameter identifier and state estimator using the cost-to-come function analysis in Section 3. In Section 4, we derive the adaptive control law based on backstepping method, and present the main results on the robustness of the system in Section 5. The effectiveness of the proposed approach is illustrated with an example in Section 6, and the paper ends with some concluding remarks in Section 7.

\section{Problem Formulation}

We consider a velocity-control problem of a brushed permanent magnet DC motor which is described by the following dynamics:

$$
\begin{aligned}
& \dot{\grave{x}}=\left[\begin{array}{cc}
-\frac{D_{l}}{J} & \frac{K_{t}}{J} \\
-\frac{K_{e}}{L} & -\frac{R}{L}
\end{array}\right] x+\left[\begin{array}{l}
0 \\
\frac{1}{L}
\end{array}\right] u+\left[\begin{array}{l}
\frac{1}{J} \\
0
\end{array}\right] T_{\check{w}}+\grave{D} \grave{w}, \\
& y=\left[\begin{array}{ll}
1 & 0
\end{array}\right] x+\grave{E} \grave{w},
\end{aligned}
$$

where $\grave{x} \in \mathbb{R}^{2}$ is the state vector and represents load shaft angular speed and motor current, respectively; $u \in \mathbb{R}$ is the scalar control input; $y \in \mathbb{R}$ is the load shaft angular speed measurement output; $L \in \mathbb{R}$ is the motor inductor; $R \in \mathbb{R}$ is the armature resistance; $K_{e}$ is the back-emf constant; $K_{t}$ is the motor torque constant; $J$ is the motor system inertia; $D_{l} \in$ $\mathbb{R}$ is the motor-system damping factor; $\grave{w}=\left[\begin{array}{lll}T_{w} & \grave{w}_{\omega} & T_{f}\end{array}\right]^{\prime}$ is the arbitrary disturbance vector, which is composed of arbitrary disturbance torque, $T_{w} \in \mathbb{R}$, arbitrary disturbance at the output measurement channel, $\grave{w}_{\omega} \in \mathbb{R}$, and friction disturbance torque $T_{f} \in \mathbb{R} ; T_{\check{w}} \in \mathbb{R}$ is the measured or estimated disturbance torque. In this paper, we assume the variables $K_{t}, K_{e}, J, D_{l}, R$, and $L$ are completely unknown or partially unknown.

It is easy to check that the true system is observable, controllable, a minimal phase system, and the transfer function from $u$ to $y$ has relative degree $r=2$. And then we can find a state diffeomorphism $x=\grave{T} \grave{x}$ and a disturbance transformation $w=\grave{M} \grave{w}$ by following [22], such that system (1a) and (1b) can be transformed into the following form in the $x$ coordinates:

$$
\begin{aligned}
& \dot{x}=A x+\left(y \bar{A}_{21}+u \bar{A}_{22}+\check{w} \bar{A}_{23}\right) \theta+B u+D w+\check{D} \check{w}, \\
& y=C x+E w,
\end{aligned}
$$

where $x \in \mathbb{R}^{2}$ is the state vector, $x=\left[\begin{array}{ll}x_{1} & x_{2}\end{array}\right]^{\prime}, \check{w}=T_{\check{w}}$, $\theta \in \mathbb{R}^{\sigma}, \sigma \in \mathbb{N}$, is the $\sigma$-dimensional vector of unknown parameters of the true system; the matrices $A, \bar{A}_{21}, \bar{A}_{22}, \bar{A}_{23}$, $D, \check{D}, C$, and $E$ are of appropriate dimensions, known and have the structure as below,

$$
\begin{aligned}
& A=\left[\begin{array}{cc}
a_{11} & 1 \\
a_{21} & a_{22}
\end{array}\right], \quad \bar{A}_{22}=\left[\begin{array}{c}
\mathbf{0}_{1 \times \sigma} \\
\bar{A}_{22} 0
\end{array}\right], \\
& B=\left[\begin{array}{c}
0 \\
b_{p 0}
\end{array}\right], \quad C=\left[\begin{array}{ll}
1 & 0
\end{array}\right],
\end{aligned}
$$

where $\bar{A}_{220}$ is a $\sigma$-dimensional row vector and $b_{p 0} \in \mathbb{R}$. Then the high-frequency gain of $(2 \mathrm{a})$ and $(2 \mathrm{~b})$ is $b_{0}=b_{p 0}+\bar{A}_{220} \theta$.

Since we consider a trajectory tracking control design problem, we make the following assumption about the reference signal $y_{d}$.

Assumption 1. The reference trajectory, $y_{d}$, is two times continuously differentiable. Define vector $Y_{d}:=\left[\begin{array}{lll}y_{d}^{(0)} & y_{d}^{(1)} & y_{d}^{(2)}\end{array}\right]^{\prime}$ and $Y_{d 0}:=\left[\begin{array}{ll}y_{d}(0) & y_{d}^{(1)}(0)\end{array}\right]^{\prime}$. The signal $Y_{d}$ is available for control design.

To guarantee the stability of the closed-loop system and the boundedness of the estimate of $\theta$, we assume there is an $a$ priori convex compact set where the parameter vector $\theta$ lies in.

Assumption 2. There exists a known smooth nonnegative radially unbounded strictly convex function $P: \mathbb{R}^{\sigma} \rightarrow \mathbb{R}$, such that the true value $\theta \in \Theta:=\left\{\bar{\theta} \in \mathbb{R}^{\sigma}: P(\bar{\theta}) \leq 1\right\}$. For all $\bar{\theta} \in \Theta, \bar{b}_{0}=b_{p 0}+\bar{A}_{22}{ }_{0} \bar{\theta}>0$.

The control objective is to design a robust adaptive controller for system (1), such that the output $y(t)$ tracks a desired reference signal $y_{d}(t)$, while rejecting the uncertainties $\left(x(0), \theta, \grave{w}_{[0, \infty)}, \check{w}_{[0, \infty)}, Y_{d 0}, y_{d}^{(2)}\right) \in \mathscr{W}:=\mathbb{R}^{2} \times \Theta \times$ $\mathscr{C} \times \mathscr{C} \times \mathbb{R}^{2} \times \mathscr{C}$ comprises the initial state, the true values of unknown parameters, unmeasured disturbances, and the measured disturbances, while all signals in the closedloop system are uniformly bounded. We make the control objective precise as follows. 
Definition 3. A controller $\mu$ is said to achieve disturbance attenuation level $\gamma$ if there exist nonnegative functions $l\left(t, \theta, x, y_{[0, t]}, T_{\check{w}[0, t]}\right)$ and $l_{0}\left(\check{x}_{0}, \check{\theta}_{0}\right)$ such that

$$
\sup _{\left(x(0), \theta, w_{\omega[0, \infty)}, T_{f[0, \infty)}, T_{w[0, \infty)}, T_{\breve{w}[0, \infty)}\right)} J^{t} \leq 0
$$

where

$$
\begin{aligned}
J^{t}= & \int_{0}^{t}\left(\left(C x-y_{d}\right)^{2}+l-\gamma^{2}\left|T_{w}\right|^{2}-\gamma^{2}\left|T_{f}\right|^{2}\right. \\
& \left.\quad-\gamma^{2}\left|w_{\omega}\right|^{2}-\gamma^{2}\left|T_{\check{w}}\right|^{2}\right) d \tau \\
& -\gamma^{2}\left|\theta-\check{\theta}_{0}\right|_{Q_{0}}^{2}-\gamma^{2}\left|x(0)-\check{x}_{0}\right|_{\Pi_{0}^{-1}}^{2}-l_{0},
\end{aligned}
$$

where $\check{\theta}_{0} \in \Theta$ is the initial guess of the unknown parameter vector; $Q_{0}>0$ is the quadratic weighting on the error between the true value of $\theta$ and the initial guess $\check{\theta}_{0}$ quantifying the level of confidence in the estimate $\check{\theta}_{0} ; \check{x}_{0}$ is the initial guess of the unknown initial state $x(0) ; \Pi_{0}^{-1}>0$ is the weighting on the initial state-estimation error, quantifying the level of confidence in the estimate $\check{x}_{0} ;|z|_{Q}$ denotes $z^{T} Q z$ for any vector $z$ and any symmetric matrix $Q$.

The control law to system (2a), (2b) is generated by the following control law

$$
u(t)=\mu\left(t, y_{[0, t]}, \check{w}_{[0, t]}\right)
$$

where $\mu:[0, \infty) \times \mathscr{L}_{2} \times \mathscr{L}_{2} \rightarrow \mathbb{R}$. We denote the class of these admissible controllers by $\mathscr{M}$.

Clearly, when the inequality (4) is achieved, we have

$$
\begin{aligned}
\left\|C x-y_{d}\right\|_{2}^{2}\left(\left\|T_{w}\right\|_{2}^{2}+\left\|T_{f}\right\|_{2}^{2}+\left\|w_{\omega}\right\|_{2}^{2}+\left\|T_{\check{w}}\right\|_{2}^{2}\right. \\
\left.+\left|\theta-\check{\theta}_{0}\right|_{Q_{0}}^{2}+\left|x(0)-\check{x}_{0}\right|_{\Pi_{0}^{-1}}^{2}+C_{0}\right)^{-1} \leq \gamma^{2},
\end{aligned}
$$

where $\|\cdot\|_{2}$ denotes $\mathscr{L}_{2}$ norm, and $C_{0}$ is a constant. When $\|\grave{w}\|_{2}$ and $\|\check{w}\|_{2}$ are finite, $\left\|C x-y_{d}\right\|_{2}$ is also finite, which implies $\lim _{t \rightarrow \infty}\left|C x-y_{d}\right|=0$, under additional assumptions.

The following notation will be used throughout this paper: $\check{x}$ denotes the estimate of the current state of the system; $\tilde{x}$ denotes the state-estimation error $x-\check{x}$; $\check{\theta}$ denotes the estimate of the parameter vector $\theta ; \widetilde{\theta}$ denotes the estimation error $\theta-\check{\theta}$; any function symbol with an "over bar" will denote a function defined in the terms of the transformed state variables, such as $\bar{f}(z)$ being the identical function as $f(x)$; for any matrix $M$, the vector $\vec{M}$ is formed by stacking up its column vectors; $e_{j, i}$ denotes a $j$-dimensional column vector, all of its elements are 0 , except its $i$ th row is 1 , such as $e_{2,2}=\left[\begin{array}{ll}0 & 1\end{array}\right]^{\prime}$.
Let $\xi$ denote the expanded state vector $\xi=\left[\begin{array}{ll}\theta^{\prime} & x^{\prime}\end{array}\right]^{\prime}$, we have the following expanded dynamics for system $(2 \mathrm{a}),(2 \mathrm{~b})$ in view of $\dot{\theta}=0$ :

$$
\begin{aligned}
\dot{\xi}= & {\left[\begin{array}{ll}
\bar{A}_{21}+u \bar{A}_{22}+\bar{A}_{23} \check{w} & A
\end{array}\right] \xi } \\
& +\left[\begin{array}{l}
\mathbf{0} \\
B
\end{array}\right] u+\left[\begin{array}{c}
\mathbf{0} \\
D
\end{array}\right] w+\left[\begin{array}{c}
\mathbf{0} \\
\check{D}
\end{array}\right] \check{w} \\
= & \bar{A} \xi+\bar{B} u+\bar{D} w+\bar{D} \check{w}, \\
y= & {\left[\begin{array}{ll}
\mathbf{0} & C
\end{array}\right] \xi+E w=: \bar{C} \xi+E w . }
\end{aligned}
$$

The worst-case optimization of the cost function (4) can be carried out in two steps as depicted in the following equations:

$$
\sup _{\left(x(0), \theta, w_{[0, \infty)}, \check{w}_{[0, \infty)}\right) \in \mathscr{W}} J^{t}=\sup _{y_{[0, \infty)}, \check{w}_{[0, \infty)}\left(x(0), \theta, w_{[0, \infty)}\right) \mid y_{[0, \infty)}, \check{w}_{[0, \infty)}} \sup ^{t} .
$$

The right-hand supremum operator will be carried out first. It is the identification design step, which will be presented in Section 3. Succinctly stated, in this step, we will calculate the maximum cost that is consistent with the given measurement waveform.

The left-hand supremum operator will be carried out second. It is the controller design step, which will be discussed in Section 4. In this step we use a backstepping method to design the control input $u$, and prove that all state variables of the closed-loop system are uniformly bounded in time for any uniformly bounded disturbance input waveforms.

This completes the formulation of the robust adaptive control problem. Next, we turn to the identification design step in the next section.

\section{Estimation Design}

In this section, we present the identification design for the adaptive control problem formulated.

In this step, the measurement waveform $y_{[0, \infty)}$ and $\check{w}_{[0, \infty)}$ are assumed to be known. Since the control input is a causal function of $y$ and $\check{w}$, then it is known. We ignore terms considered to be constant in the estimation design step, and set $l$ in (5) to be $|\xi-\widehat{\xi}|_{\overline{\mathrm{Q}}}^{2}+2(\xi-\check{\xi})^{\prime} l_{2}+\widehat{l}$. The equivalent cost function of (5) is then given by,

$$
J^{\gamma t_{f}}:=\int_{0}^{t_{f}}\left(|\xi-\widehat{\xi}|_{\overline{\mathrm{Q}}}^{2}+2(\xi-\check{\xi})^{\prime} l_{2}+\widehat{l}-\gamma^{2}|w|^{2}\right) \mathrm{d} \tau-\gamma^{2}\left|\widetilde{\xi}_{0}\right|_{\widetilde{\mathrm{Q}}_{0}}^{2},
$$

where $\overline{\widetilde{Q}}$ is a matrix-valued weighting function, $\hat{\xi}$ is the worstcase estimates for the expanded state $\xi, l_{2}$ is a design function, and $\hat{l}$ is considered to be constant in the estimation design step. The cost function is then of a linear quadratic structure, and the robust adaptive control problem becomes an $H^{\infty}$ control of affine quadratic problem, which admits a finite dimensional solution. 
We introduce the value function $W=|\xi-\check{\xi}|_{\bar{\Sigma}-1}^{2}$, and then we can obtain the dynamics of state estimator $\check{\xi}$ and worstcase covariance matrix $\bar{\Sigma}$ as below

$$
\begin{aligned}
\dot{\bar{\Sigma}}= & \left(\bar{A}-\zeta^{2} \bar{L} \bar{C}\right) \bar{\Sigma}+\bar{\Sigma}\left(\bar{A}-\zeta^{2} \bar{L} \bar{C}\right)^{\prime}+\frac{1}{\gamma^{2}} \bar{D} \bar{D}^{\prime} \\
& -\frac{1}{\gamma^{2}} \zeta^{2} \bar{L} \bar{L}^{\prime}-\bar{\Sigma}\left(\gamma^{2} \zeta^{2} \bar{C}^{\prime} \bar{C}-\bar{C}^{\prime} \bar{C}-\overline{\widetilde{Q}}\right) \bar{\Sigma}, \\
\bar{\Sigma}(0)= & \frac{1}{\gamma^{2}}\left[\begin{array}{cc}
Q_{0} & \mathbf{0} \\
\mathbf{0}_{n \times \sigma} & \Pi_{0}^{-1}
\end{array}\right]^{-1}, \\
\dot{\check{\xi}}= & \left(\bar{A}+\bar{\Sigma}\left(\bar{C}^{\prime} \bar{C}+\overline{\widetilde{Q}}\right)\right) \check{\xi}+\zeta^{2}\left(\gamma^{2} \bar{\Sigma} \bar{C}^{\prime}+\bar{L}\right)(y-\bar{C} \check{\xi}) \\
& +\bar{B} u+\bar{D} \check{w}-\bar{\Sigma}\left(\bar{C}^{\prime} y_{d}+\overline{\widetilde{Q}} \bar{\xi}\right), \\
\check{\xi}(0)= & {\left[\begin{array}{l}
\check{\theta_{0}} \\
\check{x}_{0}
\end{array}\right], }
\end{aligned}
$$

where $\zeta:=1 /\left(E E^{\prime}\right)^{1 / 2}$ and $\bar{L}$ is defined as $\bar{L}=\left[\begin{array}{ll}\mathbf{0} & L^{\prime}\end{array}\right]^{\prime}$, where $L:=D E^{\prime}$.

Then, the cost function (5) can be equivalently written as

$$
\begin{gathered}
J^{t}=-|\xi(t)-\check{\xi}(t)|_{\bar{\Sigma}-1(t)}^{2} \\
+\int_{0}^{t}\left(\left|\bar{C} \check{\xi}-y_{d}\right|^{2}+\left|\xi_{c}\right|_{\overline{\widetilde{Q}}}^{2}-\gamma^{2} \zeta^{2}|y-\bar{C} \check{\xi}|^{2}\right. \\
-\gamma^{2} \mid w-w^{*}\left(u_{[0, \tau]}, y_{[0, \tau]}, \check{w}_{[0, \tau]},\right. \\
\left.\left.\xi_{[0, \tau]}, \bar{\xi}_{[0, \tau]}, \check{\xi}_{[0, \tau]}\right)\left.\right|^{2}\right) d \tau,
\end{gathered}
$$

where $\xi_{c}:=\bar{\xi}-\check{\xi}$, which will be determined later to improve the performance of the adaptive system; $w^{*}$ is the worst-case disturbance, given by

$$
\begin{aligned}
& w^{*}\left(\tau, u_{[0, \tau]}, y_{[0, \tau]}, \check{w}_{[0, \tau]}, \xi_{[0, \tau]}, \widehat{\xi}_{[0, \tau]}, \check{\xi}_{[0, \tau]}\right) \\
& =\zeta^{2} E^{\prime}(y-\bar{C} \xi)+\frac{1}{\gamma^{2}}\left(I-\zeta^{2} E^{\prime} E\right) \bar{D}^{\prime} \bar{\Sigma}^{-1}(\xi-\check{\xi}) .
\end{aligned}
$$

We partition $\bar{\Sigma}$ as

$$
\bar{\Sigma}=\left[\begin{array}{cc}
\Sigma & \bar{\Sigma}_{12} \\
\bar{\Sigma}_{21} & \bar{\Sigma}_{22}
\end{array}\right]
$$

and introduce $\Phi:=\bar{\Sigma}_{21} \Sigma^{-1}$ and $\Pi:=\gamma^{2}\left(\bar{\Sigma}_{22}-\bar{\Sigma}_{21} \Sigma^{-1} \bar{\Sigma}_{12}\right)$.

For the boundedness of $\Sigma$, we make the following assumption on the weighting matrix $\overline{\widetilde{Q}}$.

Assumption 4. The weighting matrix $\overline{\widetilde{Q}}$ of function $l$ in (5) is given by

$$
\overline{\widetilde{Q}}=\bar{\Sigma}^{-1}\left[\begin{array}{ll}
\mathbf{0} & \mathbf{0} \\
\mathbf{0} & \Delta
\end{array}\right] \bar{\Sigma}^{-1}+\left[\begin{array}{cc}
\epsilon \Phi^{\prime} C^{\prime}\left(\gamma^{2} \zeta^{2}-1\right) C \Phi & \mathbf{0} \\
\mathbf{0} & \mathbf{0}
\end{array}\right],
$$

where $\Delta$ is $2 \times 2$ positive-definite matrix, and $\epsilon$ is a scalar function defined by

$$
\epsilon(\tau):=\frac{\operatorname{Tr}\left(\Sigma^{-1}(\tau)\right)}{K_{c}}, \quad K_{c} \geq \gamma^{2} \operatorname{Tr}\left(Q_{0}\right), \quad \tau \geq 0 .
$$
and $\Pi$ :

Then, we have the following differential equation of $\Sigma$, $\Phi$,

$$
\begin{aligned}
\dot{\Sigma}= & -(1-\epsilon) \Sigma \Phi^{\prime} C^{\prime}\left(\gamma^{2} \zeta^{2}-1\right) C \Phi \Sigma ; \\
\Sigma(0)= & \frac{1}{\gamma^{2}} Q_{0}^{-1}, \\
\dot{\Phi}= & \left(A-\zeta^{2} L C-\frac{1}{\gamma^{2}} \Pi C^{\prime}\left(\gamma^{2} \zeta^{2}-1\right) C\right) \Phi \\
& +y \bar{A}_{21}+u \bar{A}_{22}+\bar{A}_{23} \check{w} ; \quad \Phi(0)=\mathbf{0}, \\
\dot{\Pi}= & \left(A-\zeta^{2} L C\right) \Pi+\Pi\left(A-\zeta^{2} L C\right)^{\prime} \\
& -\Pi C^{\prime}\left(\zeta^{2}-\frac{1}{\gamma^{2}}\right) C \Pi \\
& +D D^{\prime}-\zeta^{2} L L^{\prime}+\gamma^{2} \Delta, \quad \Pi(0)=\Pi_{0} .
\end{aligned}
$$

The matrix $\Sigma$ will play the role of worst-case covariance matrix of the parameter estimation error. Assumption 4 guarantees that $\Sigma$ is uniformly bounded from above and uniformly bounded from below away from 0 as depicted in the following lemma, and its proof is given in [14].

Lemma 5. Consider the dynamic equation (17a) for the covariance mat-rix $\Sigma$. Let Assumption 4 hold and $\gamma \geq \zeta^{-1}$. Then, $\Sigma$ is uniformly upper and lower bounded as follows:

$$
\begin{gathered}
\frac{1}{K_{c}} \leq \Sigma(\tau) \leq \Sigma(0)=\frac{1}{\gamma^{2}} Q_{0}^{-1} ; \\
\gamma^{2} \operatorname{Tr}\left(Q_{0}\right) \leq \operatorname{Tr}\left(\Sigma^{-1}(\tau)\right) \leq K_{c} ; \quad \forall \tau \in[0, t] .
\end{gathered}
$$
by

We define $s_{\Sigma}(t):=\operatorname{Tr}\left((\Sigma(t))^{-1}\right)$, and its dynamic is given

$$
\dot{s}_{\Sigma}=\gamma^{2} \zeta^{2}(1-\epsilon) C \Phi \Phi^{\prime} C^{\prime} ; \quad s_{\Sigma}(0)=\gamma^{2} \operatorname{Tr}\left(Q_{0}\right) .
$$

Then, $\epsilon(\tau)=K_{c}^{-1} s_{\Sigma}^{-1}(\tau)$, which does not require the inversion of $\Sigma$.

From Assumption 4 and (17a), we note that $\gamma \geq \zeta^{-1}$. This means the quantity $\zeta^{-1}$ is the ultimate lower bound on the achievable performance level for the adaptive system, using the design method proposed in this paper.

Assumption 6. If the matrix $A-\zeta^{2} L C$ is Hurwitz, then the desired disturbance attenuation level $\gamma \geq \zeta^{-1}$. If the matrix $A-\zeta^{2} L C$ is not Hurwitz, then the desired disturbance attenuation level $\gamma>\zeta^{-1}$. 
Assumption 7. The initial weighting matrix $\Pi_{0}$ in $(17 \mathrm{c})$ is chosen as the unique positive definite solution to the following algebraic Riccati equation:

$$
\begin{aligned}
& \left(A-\zeta^{2} L C\right) \Pi+\Pi\left(A-\zeta^{2} L C\right)^{\prime}-\Pi C^{\prime}\left(\zeta^{2}-\frac{1}{\gamma^{2}}\right) C \Pi \\
& +D D^{\prime}-\zeta^{2} L L^{\prime}+\gamma^{2} \Delta=0 .
\end{aligned}
$$

Then, we note that the unique positive-definite solution of $(17 \mathrm{c})$ is time-invariant and equal to the initial value $\Pi_{0}$.

Remark 8. To simplify the estimator structure, we can choose $\epsilon=1$ so that $\Sigma$ will be a constant positive-definite matrix, and $s_{\Sigma}$ will be a finite positive constant. To further simplify the identifier, the initial weighting matrix $\Pi_{0}$ is chosen as the unique positive-definite solutions to its algebraic Riccati equation (17c), which also implies $\bar{\Sigma}>0$ in view of $\Sigma>0$.

To guarantee the boundedness of estimated parameters without persistently exciting signals, we introduce soft projection design on the parameter estimate. We define

$$
\rho:=\inf \left\{P(\bar{\theta}) \mid \bar{\theta} \in \mathbb{R}^{\sigma}, b_{p 0}+\bar{A}_{22}{ }_{0} \bar{\theta}=0\right\} .
$$

By Assumption 2 and Lemma 2 in [23], we have $1<\rho \leq \infty$. For any fixed $\rho_{o} \in(1, \rho)$, we define the open set

$$
\Theta_{o}:=\left\{\bar{\theta} \in \mathbb{R}^{\sigma} \mid P(\bar{\theta})<\rho_{o}\right\}
$$

Our control design will guarantee that the estimate $\check{\theta}$ lies in $\Theta_{o}$, which immediately implies $\left|b_{p 0}+\bar{A}_{220} \check{\theta}\right|>c_{0}>0$, for some $c_{0}>0$. Moreover, the convexity of $P$ implies the following inequality:

$$
\frac{\partial P}{\partial \theta}(\check{\theta})(\theta-\check{\theta})<0 \quad \forall \check{\theta} \in \mathbb{R}^{\sigma} \backslash \Theta .
$$

We set $l_{2}=\left[-\left(P_{r}(\check{\theta})\right)^{\prime} \quad \mathbf{0}\right]^{\prime}$, where

$$
P_{r}(\check{\theta}):= \begin{cases}\frac{e^{1 /(1-P(\check{\theta}))}((\partial P / \partial \theta)(\check{\theta}))^{\prime}}{\left(\rho_{o}-P(\check{\theta})\right)^{3}} & \forall \theta \in \Theta_{o} \backslash \Theta, \\ \mathbf{0} & \forall \theta \in \Theta .\end{cases}
$$

Then, we obtain

$$
\begin{aligned}
\dot{\check{\xi}}= & -\bar{\Sigma}\left[\left(P_{r}(\check{\theta})\right)^{\prime} \mathbf{0}\right]^{\prime}+\bar{A} \check{\xi}+\bar{B} u-\bar{\Sigma} \overline{\widetilde{Q}} \xi_{c} \\
& +\zeta^{2}\left(\gamma^{2} \bar{\Sigma} \bar{C}^{\prime}+\bar{L}\right)(y-\bar{C} \check{\xi})+\overline{\check{D}} \check{w}, \\
\check{\xi}(0)= & {\left[\begin{array}{ll}
\check{\theta}_{0}^{\prime} & \check{x}_{0}^{\prime}
\end{array}\right]^{\prime}, }
\end{aligned}
$$

where $\xi_{c}=\widehat{\xi}-\check{\xi}$.
We summarize the estimation dynamics equations below

$$
\begin{gathered}
\left(A-\zeta^{2} L C\right) \Pi+\Pi\left(A-\zeta^{2} L C\right) \\
-\Pi C^{\prime}\left(\zeta^{2}-\frac{1}{\gamma^{2}}\right) C \Pi+D D^{\prime}-\zeta^{2} L L^{\prime}+\gamma^{2} \Delta=0 \\
\dot{\Sigma}=-(1-\epsilon) \Sigma \Phi^{\prime} C^{\prime}\left(\gamma^{2} \zeta^{2}-1\right) C \Phi \Sigma ; \quad \Sigma(0)=\frac{1}{\gamma^{2}} Q_{0}^{-1},
\end{gathered}
$$

$$
\dot{s}_{\Sigma}=\left(\gamma^{2} \zeta^{2}-1\right)(1-\epsilon) C \Phi \Phi^{\prime} C^{\prime} ; \quad s_{\sigma}(0)=\gamma^{2} \operatorname{Tr}\left(Q_{0}\right)
$$

$$
\epsilon=\frac{1}{K_{c} s_{\Sigma}}
$$

$$
A_{f}=A-\zeta^{2} L C-\Pi C^{\prime} C\left(\zeta^{2}-\frac{1}{\gamma^{2}}\right) \text {, }
$$

$$
\dot{\Phi}=A_{f} \Phi+y \bar{A}_{21}+u \bar{A}_{22}+\check{w} \bar{A}_{23} ; \quad \Phi(0)=\mathbf{0},
$$

$$
\begin{aligned}
\dot{\theta}= & -\Sigma P_{r}(\check{\theta})-\Sigma \Phi^{\prime} C^{\prime}\left(y_{d}-C \check{x}\right) \\
& -\left[\Sigma \Sigma \Phi^{\prime}\right] \overline{\widetilde{Q}} \xi_{c}+\gamma^{2} \zeta^{2} \Sigma \Phi^{\prime} C^{\prime}(y-C \check{x}) ; \quad \check{\theta}(0)=\check{\theta}_{0},
\end{aligned}
$$

$$
\begin{aligned}
\check{\check{x}}= & -\Phi \sum P_{r}(\check{\theta})+A \check{x}-\left(\frac{1}{\gamma^{2}} \Pi+\Phi \Sigma \Phi^{\prime}\right) C^{\prime}\left(y_{d}-C \check{x}\right) \\
& -\left[\Phi \Sigma \frac{1}{\gamma^{2}} \Pi+\Phi \Sigma \Phi^{\prime}\right] \overline{\widetilde{Q}} \xi_{c} \\
& +\left(y \bar{A}_{21}+u \bar{A}_{22}+\check{w} \bar{A}_{23}\right) \check{\theta} \\
& +\zeta^{2}\left(\Pi C^{\prime}+\gamma^{2} \Phi \Sigma \Phi^{\prime} C^{\prime}+L\right) \\
& \times(y-C \check{x})+\check{D} \check{w}+B u ; \quad \check{x}(0)=\check{x}_{0} .
\end{aligned}
$$

For the controller structure simplification, the dynamics for $\Phi$ can be implemented as below. First, we observe the matrix $A_{f}$ has the same structure as the matrix $A$. Then, we introduce the matrix

$$
M_{f}:=\left[\begin{array}{ll}
A_{f} p_{2} & p_{2}
\end{array}\right],
$$

where $p_{2}$ is a 2-dimensional vector such that the pair $\left(A_{f}, p_{2}\right)$ is controllable, which implies that $M_{f}$ is invertible. Then the following prefiltering system for $y, u$, and $\check{w}$ generates the $\Phi$ online:

$$
\begin{aligned}
\dot{\eta}= & A_{f} \eta+p_{2} y ; \quad \eta(0)=0, \\
\dot{\lambda}= & A_{f} \lambda+p_{2} u ; \quad \lambda(0)=0, \\
\dot{\eta}^{\check{w}}= & A_{f} \eta^{\check{w}}+p_{2} \check{w} ; \quad \eta^{\check{w}}(0)=0, \\
\Phi= & {\left[\begin{array}{ll}
A_{f} \eta & \eta
\end{array}\right] M_{f}^{-1} \bar{A}_{21}+\left[\begin{array}{ll}
A_{f} \lambda & \lambda
\end{array}\right] M_{f}^{-1} \bar{A}_{22} } \\
& +\left[\begin{array}{lll}
A_{f} \eta^{\check{w}} & \eta^{\check{w}}
\end{array}\right] M_{f}^{-1} \bar{A}_{23} .
\end{aligned}
$$


Associated with the above identifier, introduce the value function

$$
\begin{aligned}
& W(t, \xi(t), \check{\xi}(t), \bar{\Sigma}(t)) \\
& \quad=|\xi(t)-\check{\xi}(t)|_{\bar{\Sigma}^{-1}(t)}^{2} \\
& \quad=|\theta-\check{\theta}(t)|_{\Sigma^{-1}(t)}^{2}+\gamma^{2}|x(t)-\check{x}(t)-\Phi(t)(\theta-\check{\theta}(t))|_{\Pi^{-1}}^{2}
\end{aligned}
$$

whose time derivative is given by

$$
\begin{aligned}
\dot{W}= & -\left|C x-y_{d}\right|^{2}-\gamma^{4}|x-\widehat{x}-\Phi(\theta-\widehat{\theta})|_{\Pi^{-1} \Delta \Pi^{-1}}^{2} \\
& -\epsilon\left(\gamma^{2} \zeta^{2}-1\right)|\theta-\widehat{\theta}|_{\Phi^{\prime} C^{\prime} C \Phi}^{2}+\gamma^{2}|w|^{2} \\
& +\left|C \check{x}-y_{d}\right|^{2}+\left|\xi_{c}\right|_{\overline{\mathrm{Q}}}^{2}-\gamma^{2} \zeta^{2}|y-C \check{x}|^{2} \\
& -\gamma^{2}\left|w-w^{*}\left(t, u_{[0, t]}, y_{[0, t]}, \check{w}_{[0, t]}, \xi_{[0, t]}, \widehat{\xi}_{[0, t]}, \check{\xi}_{[0, t]}\right)\right|^{2} \\
& +2(\theta-\check{\theta})^{\prime} P_{r}(\check{\theta}) .
\end{aligned}
$$

We note that the last term in $\dot{W}$ is nonpositive, zero on the set $\Theta$ and approaches $-\infty$ as $\check{\theta}$ approaches the boundary of the set $\Theta$, which guarantees the boundness of $\check{\theta}$.

Then the cost function can be equivalently written as

$$
\begin{aligned}
J^{t}= & J^{t}+W(0)-W(t)+\int_{0}^{t} \dot{W} d \tau \\
= & -|\theta-\check{\theta}(t)|_{\Sigma^{-1}(t)}^{2}-\gamma^{2}|x(t)-\check{x}(t)-\Phi(t)(\theta-\check{\theta}(t))|_{\Pi^{-1}}^{2} \\
& +\int_{0}^{t}\left(\left|C \check{x}-y_{d}\right|^{2}+\left|\xi_{c}\right| \frac{2}{\widetilde{Q}}-\gamma^{2} \zeta^{2}|y-C \check{x}|^{2}-\gamma^{2} \mid w-w^{*}\right. \\
& \times\left.\left(\tau, u_{[0, \tau]}, y_{[0, \tau]}, \check{w}_{[0, \tau]}, \xi_{[0, \tau]}, \widehat{\xi}_{[0, \tau]} \check{\xi}_{[0, \tau]}\right)\right|^{2} \\
& \left.+\widehat{l}-\gamma^{2}|\check{w}|^{2}\right) d \tau .
\end{aligned}
$$

This completes the identification design step.

\section{Control Design}

In this section, we describe the controller design for the uncertain system under consideration. Note that, we ignored some terms in the cost function (5) in the identification step, since they are constant when $y$ and $\check{w}$ are given. In the control design step, we will include such terms. Then, based on the cost function (5) in the Section 2, the controller design is to guarantee that the following supremum is less than or equal to zero for all measurement waveforms:

$$
\begin{gathered}
\sup _{\left(x(0), \theta, w_{[0, \infty)} \check{w}_{[0, \infty)}\right) \in \mathscr{W}} J^{t} \\
\leq \sup _{y_{[0, \infty)}, \check{w}_{[0, \infty)}}\left\{\int _ { 0 } ^ { t } \left(\left|C \check{x}-y_{d}\right|^{2}+\left|\xi_{c}\right|^{2} \overline{\widetilde{Q}}-\gamma^{2} \zeta^{2}|y-C \check{x}|^{2}\right.\right. \\
\left.\left.+\widehat{l}-\gamma^{2}|\check{w}|^{2}\right) d \tau-l_{0}\right\},
\end{gathered}
$$

where function $\widehat{l}\left(\tau, y_{[0, \tau]}, \check{w}_{[0, \tau]}\right)$ is part of the weighting function $l\left(\tau, \theta, x, y_{[0, \tau]}, \check{w}_{[0, \tau]}\right)$ to be designed, which is a constant in the identifier design step, and is therefore neglected.

By (32), we observe that the cost function is expressed in term of the states of the estimator we derived, whose dynamics are driven by the measurement $y$, input $u$, measured disturbance $\check{w}$, and the worst-case estimate for the expanded state vector $\widehat{\xi}$, which are signals we either measure or can construct. This is then a nonlinear $H^{\infty}$-optimal control problem under full information measurements. Instead of considering $y$ and $\check{w}$ as the maximizing variable, we can equivalently deal with the transformed variable

$$
v:=\left[\begin{array}{c}
\zeta(y-C \check{x}) \\
\check{w}
\end{array}\right] .
$$

Then, we have

$$
\begin{aligned}
\dot{\eta}= & A_{f} \eta+p_{2} C \check{x}+p_{2}\left(\frac{e_{2,1}^{\prime} v}{\zeta}\right) \\
\dot{\theta}= & -\Sigma P_{r}(\check{\theta})-\Sigma \Phi^{\prime} C^{\prime}\left(y_{d}-C \check{x}\right) \\
& -\left[\Sigma \Sigma \Phi^{\prime}\right] \overline{\widetilde{Q}} \xi_{c}+\gamma^{2} \Sigma \Phi^{\prime} C^{\prime} \zeta e_{2,1}^{\prime} v \\
\dot{\check{x}}= & A \check{x}-\left(\frac{1}{\gamma^{2}} \Pi+\Phi \Sigma \Phi^{\prime}\right) C^{\prime}\left(y_{d}-C \check{x}\right)+\bar{A}_{21} \check{\theta} C \check{x} \\
& -\Phi \Sigma P_{r}(\check{\theta})-\left[\Phi \Sigma \frac{1}{\gamma^{2}} \Pi+\Phi \Sigma \Phi^{\prime}\right] \overline{\widetilde{Q}}_{c}+B u \\
& +\bar{A}_{22} \check{\theta} u+\left(\left(\zeta^{-2} \bar{A}_{21} \check{\theta}+\Pi C^{\prime}+\gamma^{2} \Phi \Sigma \Phi^{\prime} C^{\prime}+L\right) \zeta e_{2,1}^{\prime}\right. \\
& \left.+\bar{A}_{23} \check{\theta}_{2,2}^{\prime}+\left[\mathbf{0}_{n \times 1} \check{D}\right]\right) v
\end{aligned}
$$

$$
\begin{aligned}
\dot{W}= & -\left|C x-y_{d}\right|^{2}-\gamma^{4}|x-\widehat{x}-\Phi(\theta-\widehat{\theta})|_{\Pi^{-1} \Delta \Pi^{-1}}^{2} \\
& -\epsilon\left(\gamma^{2} \zeta^{2}-1\right)|\theta-\widehat{\theta}|_{\Phi^{\prime} C^{\prime} C \Phi}^{2} \\
& +\left|C \check{x}-y_{d}\right|^{2}+\left|\xi_{c}\right|_{\overline{\widetilde{Q}}}^{2}+2(\theta-\check{\theta})^{\prime} P_{r}(\check{\theta}) \\
& +\gamma^{2}|\check{w}|^{2}+\gamma^{2}|w|^{2}-\gamma^{2}|v|^{2} \\
& -\gamma^{2}\left|w-w^{*}\left(t, u_{[0, t]}, y_{[0, t]}, \check{w}_{[0, t]}, \xi_{[0, t]}, \widehat{\xi}_{[0, t]}, \check{\xi}_{[0, t]}\right)\right|^{2} .
\end{aligned}
$$


The variables to be designed at this stage include $u$ and $\xi_{c}$. The design for $\xi_{c}$ will be carried out last. Note that the structure of $A$ in the dynamics is in strict-feedback form, we will use the backstepping methodology [24] to design the control input $u$, which will guarantee the global uniform boundedness of the closed-loop system states and the asymptotic convergence of tracking error.

Consider the dynamics of $\Phi$,

$$
\dot{\Phi}=A_{f} \Phi+y \bar{A}_{21}+u \bar{A}_{22}+\check{w} \bar{A}_{23} ; \quad \Phi(0)=\mathbf{0} .
$$

For ease of the ensuing study, we will separate $\Phi$ as the sum of several matrices as follows:

$$
\begin{aligned}
& \Phi=\Phi^{u}+\Phi^{y}+\Phi^{\check{w}}, \\
& \Phi^{y}=\left[\begin{array}{ll}
A_{f} \eta & \eta
\end{array}\right] M_{f}^{-1} \bar{A}_{21}=\left[\begin{array}{c}
\eta^{\prime} T_{1} \\
\eta^{\prime} T_{2}
\end{array}\right], \\
& \dot{\Phi}^{u}=A_{f} \Phi^{u}+u \bar{A}_{22} ; \quad \Phi^{u}(0)=\mathbf{0}, \\
& \dot{\Phi}^{\check{w}}=A_{f} \Phi^{\check{w}}+\check{w} \bar{A}_{23} ; \quad \Phi^{\check{w}}(0)=\mathbf{0},
\end{aligned}
$$

where $T_{i}, i=1,2$ are $2 \times 1$-dimensional constant matrices depending on $A_{f}, M_{f}$, and $\bar{A}_{21}$. Express $\Phi^{u}$ and $\Phi^{\check{w}}$ in terms of their row vectors, $\Phi^{u}=\left[\begin{array}{ll}\Phi_{1}^{u^{\prime}} & \Phi_{2}^{u^{\prime}}{ }^{\prime}\end{array}\right]^{\prime}$ and $\Phi^{\check{w}}=\left[\begin{array}{ll}\Phi_{1}^{\check{w} \prime} & \Phi_{2}^{\check{w} \prime}\end{array}\right]^{\prime}$. Then, $C \Phi^{y}=\eta^{\prime} T_{1}, C \Phi^{u}=\Phi_{1}^{u}$, and $C \Phi^{\check{w}}=\Phi_{1}^{\check{w}}$.

We summarized the dynamics for backstepping design in the following, where we have emphasized the dependence of various functions on the independent variables:

$$
\begin{aligned}
& \dot{s}_{\Sigma}=\left(\gamma^{2} \zeta^{2}-1\right)(1-\epsilon)\left(\eta^{\prime} T_{1}+\Phi_{1}^{u}+\Phi_{1}^{\check{w}}\right) \\
& \times\left(\eta^{\prime} T_{1}+\Phi_{1}^{u}+\Phi_{1}^{\check{w}}\right)^{\prime}, \\
& \epsilon=\frac{1}{K_{c} s_{\Sigma}}, \\
& \dot{\Sigma}=-(1-\epsilon) \Sigma\left(\eta^{\prime} T_{1}+\Phi_{1}^{u}+\Phi_{1}^{\check{w}}\right)^{\prime} \\
& \times\left(\gamma^{2} \zeta^{2}-1\right)\left(\eta^{\prime} T_{1}+\Phi_{1}^{u}+\Phi_{1}^{\check{w}}\right) \Sigma, \\
& \dot{\theta}=\delta\left(y_{d}-\check{x}_{1}, \eta, \Phi_{1}^{\check{w}}, \Phi_{1}^{u}, \check{\theta}, \vec{\Sigma}\right) \\
&+\varphi\left(\eta, \vec{\Phi}^{\check{w}}, \vec{\Phi}^{u}, \vec{\Sigma}\right) \overline{\widetilde{Q}} \xi_{c}+\kappa\left(\eta, \Phi_{1}^{\check{w}}, \Phi_{1}^{u}, \vec{\Sigma}\right) v, \\
& \quad \dot{\eta}=A_{f} \eta+p_{2} \check{x}_{1}+p_{2}\left(\frac{e_{2,1}^{\prime} v}{\zeta}\right), \\
& \check{x}_{1}=\check{x}_{2}+f_{1}\left(y_{d}-\check{x}_{1}, \check{x}_{1}, \check{\theta}, \eta, \Phi_{1}^{\check{w}}, \Phi_{1}^{u}, \vec{\Sigma}\right) \\
&+\varrho_{1}\left(\eta, \breve{\Phi}^{\check{w}}, \vec{\Phi}^{u}, \vec{\Sigma}\right) \overline{\widetilde{Q}} \xi_{c} \\
&+h_{1}\left(\check{\theta}, \eta, \Phi_{1}^{\check{w}}, \Phi_{1}^{u}, \vec{\Sigma}\right) v,
\end{aligned}
$$

$$
\begin{aligned}
& \dot{x}_{2}= a_{22} \check{x}_{2}+\left(b_{p 0}+\bar{A}_{220} \check{\theta}\right) u \\
&+f_{2}\left(y_{d}-\check{x}_{1}, \check{x}_{1}, \check{x}_{2}, \check{\theta}, \eta, \Phi_{1}^{\check{w}}, \Phi_{2}^{\check{w}}, \Phi_{1}^{u}, \Phi_{2}^{u}, \vec{\Sigma}\right) \\
&+\varrho_{2}\left(\eta, \vec{\Phi}^{\check{w}}, \vec{\Phi}^{u}, \vec{\Sigma}\right) \overline{\widetilde{Q}} \xi_{c} \\
&+h_{2}\left(\check{\theta}, \eta, \Phi_{1}^{\check{w}}, \Phi_{2}^{\check{w}}, \Phi_{1}^{u}, \Phi_{2}^{u}, \vec{\Sigma}\right) v, \\
& \dot{\Phi}_{1}^{u}=\psi_{1}\left(\Phi_{1}^{u}\right)+\Phi_{2}^{u}, \\
& \dot{\Phi}_{1}^{\check{w}}=\Phi_{1}\left(\Phi_{1}^{\check{w}}\right)+\Phi_{2}^{\check{w}}+e_{2,1}^{\prime} \bar{A}_{23} e_{2,2}^{\prime} v,
\end{aligned}
$$

where the nonlinear functions $\delta, f_{1}$, and $f_{2}$ are smooth as long as $\check{\theta} \in \Theta_{o}$; the nonlinear functions $\varphi, \kappa, \varrho_{1}, \varrho_{2}, h_{1}, h_{2}$, $\psi_{1}$, and $\omega_{1}$ are smooth. Here, we use $\Phi_{1}^{u}, \Phi_{2}^{u}, \Phi_{1}^{\check{\omega}}$, and $\Phi_{2}^{\check{\omega}}$ as independent variables, instead of $\lambda, \eta_{1}^{\check{w}}$, for the clarity of ensuing analysis.

We observe that the above dynamics is linear in $\xi_{c}$, which will be optimatized after backstepping design. $\Sigma, s_{\Sigma}, \Phi^{\check{\omega}}$, and $\check{\theta}$ will always be bounded by the design in Section 3, then they will not be stabilized in the control design. $\Phi^{u}$ is not necessary bounded, since the control input $u$ appeared in their dynamics, it can not stabilzed in conjunction with $\check{x}$ using backstepping. Hence, we assume it is bounded, and prove later that it is indeed so under the derived control law.

The following backstepping design will achieve the $\gamma$ level of disturbance attenuation with respect to the disturbance $v$.

Step 1. In this step, we try to stabilize $\eta$ by virtual control law $\check{x}_{1}=y_{d}$. Introduce variable $\eta_{d}$, as the desired trajectory of $\eta$, which satisfies the dynamics

$$
\dot{\eta}_{d}=A_{f} \eta_{d}+p_{2} y_{d} ; \quad \eta_{d}(0)=\mathbf{0}_{2 \times 1} .
$$

Define the error variable $\widetilde{\eta}=\eta-\eta_{d}$. Then, $\widetilde{\eta}$ satisfies the dynamics

$$
\dot{\tilde{\eta}}=A_{f} \widetilde{\eta}+p_{2}\left(\frac{e_{2,1}^{\prime} v}{\zeta}\right)+p_{2}\left(\check{x}_{1}-y_{d}\right) .
$$

By [14], the following holds.

Lemma 9. Given any Hurwitz matrix $A_{f}$, there exists a positive-definite matrix $Y$ such that the following generalized algebraic Riccati equation admits a positive-definite solution Z:

$$
A_{f}^{\prime} Z+Z A_{f}+\frac{1}{\gamma^{2} \zeta^{2}} Z p_{2} p_{2}^{\prime} Z+Y=0 .
$$

Note that $A_{f}$ in (42) is a Hurwitz matrix, then we define the following value function in terms of the positive-definite matrix $Z$ :

$$
V_{0}(\widetilde{\eta}):=|\widetilde{\eta}|_{Z}^{2}
$$

Then its time derivative is given by

$$
\dot{V}_{0}=-|\tilde{\eta}|_{Y}^{2}+\gamma^{2}|v|^{2}-\gamma^{2}\left|v-v_{0}\right|^{2}+2 \widetilde{\eta}^{\prime} Z p_{n}\left(\check{x}_{1}-y_{d}\right),
$$


where

$$
v_{0}(\widetilde{\eta})=\frac{1}{\gamma^{2} \zeta} e_{2,1} p_{2}^{\prime} Z \widetilde{\eta} .
$$

If $\check{x}_{1}$ is control input, then we may choose the control law

$$
\check{x}_{1}=y_{d}
$$

and the design achieves attenuation level $\gamma$ from the disturbance $v$ to the output $Y^{1 / 2}\left(\eta-\eta_{d}\right)$. This completes the virtual control design for the $\eta$ dynamics.

Step 2. Define the transformed variable

$$
z_{1}:=\check{x}_{1}-y_{d}
$$

which is the deviation of $\check{x}_{1}$ from its desired trajectory $y_{d}$.

Then the time derivative of $z_{1}$ is given by

$$
\begin{aligned}
\dot{z}_{1}= & \bar{f}_{1}\left(z_{1}, y_{d}, \check{\theta}, \eta, \Phi_{1}^{\check{w}}, \Phi_{1}^{u}, \vec{\Sigma}\right)+\check{x}_{2}-y_{d}^{(1)} \\
& +\varrho_{1}\left(\eta, \breve{\Phi}^{\check{w}}, \vec{\Phi}^{u}, \vec{\Sigma}\right) \overline{\widetilde{Q}} \xi_{c}+h_{1}\left(\check{\theta}, \eta, \Phi_{1}^{\check{\omega}}, \Phi_{1}^{u}, \vec{\Sigma}\right) v
\end{aligned}
$$

where the function $\bar{f}_{1}$ is defined as

$$
\begin{aligned}
& \bar{f}_{1}\left(z_{1}, y_{d}, \check{\theta}, \eta, \Phi_{1}^{\check{\omega}}, \Phi_{1}^{u}, \vec{\Sigma}\right) \\
& \quad=f_{1}\left(y_{d}-\check{x}_{1}, \check{x}_{1}, \check{\theta}, \eta, \Phi_{1}^{\check{\omega}}, \Phi_{1}^{u}, \vec{\Sigma}\right) .
\end{aligned}
$$

Introduce the value function for this step

$$
\bar{V}_{1}=V_{0}+\frac{1}{2} z_{1}^{2}
$$

whose derivative is given by

$$
\begin{aligned}
\dot{\bar{V}}_{1}= & -|\widetilde{\eta}|_{Y}^{2}+\gamma^{2}|v|^{2}-\gamma^{2}\left|v-v_{0}\right|^{2}+2 \widetilde{\eta} Z p_{n} z_{1} \\
& +z_{1}\left(\check{x}_{2}-y_{d}^{(1)}+\bar{f}_{1}+\varrho_{1} \overline{\widetilde{Q}} \xi_{c}+h_{1} v\right) \\
= & -z_{1}^{2}-|\widetilde{\eta}|_{Y}^{2}-\bar{\beta}_{1} z_{1}^{2}+z_{1} z_{2}+\bar{\zeta}_{1}^{\prime} \overline{\widetilde{Q}} \xi_{c} \\
& +\gamma^{2}|v|^{2}-\gamma^{2}\left|v-\bar{v}_{1}\right|^{2},
\end{aligned}
$$

where

$$
\begin{aligned}
& z_{2}=\check{x}_{2}-y_{d}^{(1)}-\bar{\alpha}_{1} \text {, } \\
& \bar{v}_{1}\left(z_{1}, \check{\theta}, \widetilde{\eta}, \eta, \Phi_{1}^{\check{w}}, \Phi_{1}^{u}, \vec{\Sigma}\right)=v_{0}+\frac{1}{2 \gamma^{2}} h_{1}^{\prime} z_{1}, \\
& \bar{\alpha}_{1}\left(z_{1}, y_{d}, \check{\theta}, \widetilde{\eta}, \eta, \Phi_{1}^{\check{w}}, \Phi_{1}^{u}, \vec{\Sigma}, s\right)=-z_{1}-\bar{\beta}_{1} z_{1}-2 p_{n}^{\prime} Z \widetilde{\eta} \\
& -\bar{f}_{1}-h_{1} v_{0}-\frac{1}{4 \gamma^{2}} h_{1} h_{1}^{\prime} z_{1} \text {, } \\
& \bar{\beta}_{1}\left(z_{1}, y_{d}, \check{\theta}, \widetilde{\eta}, \eta, \Phi_{1}^{\check{\omega}}, \Phi_{1}^{u}, \vec{\Sigma}, s\right) \geq c_{\beta_{1}}>0, \\
& \bar{\varsigma}_{1}\left(z_{1}, y_{d}, \widetilde{\eta}, \eta, \Phi_{1}^{\check{w}}, \Phi^{u}, \vec{\Sigma}\right)=\varrho_{1}^{\prime} z_{1},
\end{aligned}
$$

where $c_{\beta_{1}}$ is any positive constant, and the nonlinear function $\bar{\beta}_{1}$ is to be chosen by the designer. Note that the function $\alpha_{1}$ is smooth as long as $\check{\theta} \in \Theta_{o}$. If $\check{x}_{2}$ were the actual controls, then we would choose the following control law:

$$
\check{x}_{2}=y_{d}^{(1)}+\bar{\alpha}_{1} \text {, }
$$

and set $\xi_{c}=0$, to guarantee the dissipation inequality with supply rate

$$
-\left|\check{x}_{1}-y_{d}\right|^{2}-|\tilde{\eta}|_{Y}^{2}-\bar{\beta}_{1} z_{1}^{2}+\gamma^{2} v^{2}
$$

This completes the second step of backstepping design.

Step 3. At this step the actual control appears in the derivative of $z_{2}$, which is given by

$$
\begin{aligned}
\dot{z}_{2}= & a_{22} \check{x}_{2}+\left(b_{p 0}+\bar{A}_{220} \check{\theta}\right) u \\
& -y_{d}^{(2)}+\bar{\chi}_{21}+2 \gamma^{2} \bar{\chi}_{22} v+\bar{\chi}_{23} \overline{\widetilde{Q}} \xi_{c},
\end{aligned}
$$

where $\chi_{21}, \chi_{22}$, and $\chi_{23}$ are given as follows:

$$
\begin{aligned}
\chi_{21}= & f_{2}-\frac{\partial \alpha_{1}}{\partial \check{x}_{1}}\left(f_{1}+\check{x}_{2}\right)-\frac{\partial \alpha_{1}}{\partial y_{d}} y_{d}^{(1)} \\
& -\frac{\partial \alpha_{1}}{\partial \check{\theta}} \delta-\frac{\partial \alpha_{1}}{\partial \tilde{\eta}}\left(A_{f} \tilde{\eta}+p_{2} z_{1}\right) \\
& -\frac{\partial \alpha_{1}}{\partial \eta}\left(A_{f} \eta+p_{2} \check{x}_{1}\right)-\frac{\partial \alpha_{1}}{\partial \Phi_{1}^{\check{\omega}}}\left(\Phi_{2}^{\check{\omega}}+\Phi_{1}\right)^{\prime} \\
& -\frac{\partial \alpha_{1}}{\partial \Phi_{1}^{u}}\left(\Phi_{2}^{u}+\psi_{1}\right)^{\prime}-\frac{\partial \alpha_{1}}{\partial \vec{\Sigma}}(\epsilon-1) \\
& \times\left(\Sigma\left(\eta^{\prime} T_{1}+\Phi_{1}^{\check{\omega}}+\Phi_{1}^{u}\right)^{\prime}\left(\gamma^{2} \zeta^{2}-1\right)\left(\eta^{\prime} T_{1}+\Phi_{1}^{\check{\omega}}+\Phi_{1}^{u}\right) \Sigma\right) \\
& -\frac{\partial \alpha_{1}}{\partial s_{\Sigma}}\left(\gamma^{2} \zeta^{2}-1\right)(1-\epsilon)\left(\eta^{\prime} T_{1}+\Phi_{1}^{\check{\omega}}+\Phi_{1}^{u}\right) \\
& \times\left(\eta^{\prime} T_{1}+\Phi_{1}^{\check{\omega}}+\Phi_{1}^{u}\right)^{\prime}, \\
\chi_{22}= & \frac{1}{2 \gamma^{2}}\left(h_{2}-\frac{\partial \alpha_{1}}{\partial \check{x}_{1}} h_{1}-\frac{\partial \alpha_{1}}{\partial \check{\theta}} \kappa-\frac{\partial \alpha_{1}}{\partial \widetilde{\eta}} \frac{p_{2} e_{2,1}^{\prime}}{\zeta}\right. \\
& \left.\quad-\frac{\partial \alpha_{1}}{\partial \eta} \frac{p_{2} e_{2,1}^{\prime}}{\zeta}-\frac{\partial \alpha_{1}}{\partial \Phi_{1}^{\check{\omega}}} \bar{A}_{23}^{\prime} e_{2,2} e_{2,2}^{\prime}\right), \\
\chi_{23}= & \varrho_{2}-\frac{\partial \alpha_{1}}{\partial \check{x}_{1}} \varrho_{1}-\frac{\partial \alpha_{1}}{\partial \check{\theta}} \varphi .
\end{aligned}
$$

Introduce the following value function for this step:

$$
\bar{V}_{2}=\bar{V}_{1}+\frac{1}{2} z_{2}^{2}
$$

Its derivative can be written as

$$
\dot{\bar{V}}_{2}=-z_{1}^{2}-|\tilde{\eta}|_{Y}^{2}-\sum_{j=1}^{2} \bar{\beta}_{j} z_{j}^{2}+\gamma^{2}|v|^{2}-\gamma^{2}\left|v-\bar{v}_{2}\right|^{2}+\bar{\zeta}_{2}^{\prime} \overline{\widetilde{Q}} \xi_{c},
$$


with the control law defined by

$$
\begin{aligned}
& u=\bar{\mu}\left(z_{1}, z_{2}, \check{x}_{1}, \check{x}_{2}, y_{d}, y_{d}^{(1)},\right. \\
& \left.\check{\theta}, \tilde{\eta}, \eta, \Phi_{1}^{\check{w}}, \Phi_{2}^{\check{w}}, \Phi_{1}^{u}, \ldots, \Phi_{r}^{u}, \check{w}, \vec{\Sigma}, s_{\Sigma}\right) \\
& :=-\frac{1}{b_{p 0}+\bar{A}_{220} \check{\theta}}\left(a_{22} \check{x}_{2}-y_{d}^{(2)}-\bar{\alpha}_{2}\right),
\end{aligned}
$$

where

$$
\begin{gathered}
\bar{\alpha}_{2}=-\bar{\chi}_{21}-2 \gamma^{2} \bar{\chi}_{22,2} \check{w}-2 \gamma^{2} \bar{\chi}_{22,1} e_{2,1}^{\prime} \bar{v}_{1} \\
-\gamma^{2} \bar{\chi}_{22,1}^{2} z_{2}-\bar{\beta}_{2} z_{2}-z_{1}, \\
\bar{v}_{2}=\bar{v}_{1}+e_{2,1} \bar{\chi}_{22,1} z_{2},
\end{gathered}
$$

where $\bar{\chi}_{22}=\left[\begin{array}{ll}\bar{\chi}_{22,1} & \bar{\chi}_{22,2}\end{array}\right]$. Clearly, the functions $\bar{\mu}, \bar{\chi}_{21}, \bar{\chi}_{22}$, $\bar{\chi}_{23}, \bar{v}_{2}$, and $\bar{\varsigma}_{2}$ are smooth as long as $\check{\theta} \in \Theta_{o}$.

This completes the backstepping design procedure.

For the closed-loop adaptive nonlinear system, we have the following value function:

$$
\begin{aligned}
U:= & V_{2}+W=|\theta-\check{\theta}|_{\Sigma^{-1}}^{2}+\gamma^{2}|x-\check{x}-\Phi(\theta-\check{\theta})|_{\Pi^{-1}}^{2} \\
& +|\tilde{\eta}|_{Z}^{2}+\frac{1}{2} \sum_{j=1}^{2}\left(\check{x}_{j}-y_{d}^{(j-1)}-\alpha_{j-1}\right)^{2},
\end{aligned}
$$

where we have introduced $\alpha_{0}=0$ for notational consistency. The time derivative of this function is given by

$$
\begin{aligned}
\dot{U}= & -\left|C x-y_{d}\right|^{2}-\gamma^{4}|x-\widehat{x}-\Phi(\theta-\widehat{\theta})|_{\Pi^{-1} \Delta \Pi^{-1}}^{2} \\
& -\epsilon\left(\gamma^{2} \zeta^{2}-1\right)|\theta-\widehat{\theta}|_{\Phi^{\prime} C^{\prime} C \Phi}^{2} \\
& +2(\theta-\check{\theta})^{\prime} P_{r}(\check{\theta})+\left|\xi_{c}\right|_{\overline{\mathrm{Q}}}^{2}+\bar{\zeta}_{r}^{\prime} \overline{\widetilde{Q}} \xi_{c}-|\widetilde{\eta}|_{Y}^{2} \\
& -\sum_{j=1}^{2} \bar{\beta}_{j} z_{j}^{2}+\gamma^{2}|w|^{2}+\gamma^{2}|\check{w}|^{2} \\
& -\gamma^{2}\left|w-w^{*}\left(t, u_{[0, t]}, y_{[0, t]}, \check{w}_{[0, t]}, \xi_{[0, t]}, \widehat{\xi}_{[0, t]}, \check{\xi}_{[0, t]}\right)\right|^{2} \\
& -\gamma^{2}\left|v-\bar{v}_{2}\right|^{2} \\
= & -\left|C x-y_{d}\right|^{2}-\gamma^{4}|x-\widehat{x}-\Phi(\theta-\widehat{\theta})|_{\Pi^{-1} \Delta \Pi^{-1}}^{2} \\
& -\epsilon\left(\gamma^{2} \zeta^{2}-1\right)|\theta-\widehat{\theta}|_{\Phi^{\prime} C^{\prime} C \Phi}^{2} \\
& +2(\theta-\check{\theta})^{\prime} P_{r}(\check{\theta})+\left|\xi_{c}+\frac{1}{2} \bar{\varsigma}_{2}\right|_{\overline{\widetilde{Q}}}^{2}-\frac{1}{4}\left|\bar{\zeta}_{2}\right|_{\widetilde{\mathrm{Q}}_{\overline{\mathrm{Q}}}^{2}}
\end{aligned}
$$

$$
\begin{aligned}
& -|\widetilde{\eta}|_{Y}^{2}-\sum_{j=1}^{2} \bar{\beta}_{j} z_{j}^{2}+\gamma^{2}|w|^{2}+\gamma^{2}|\check{w}|^{2} \\
& -\gamma^{2}\left|w-w^{*}\left(t, u_{[0, t]}, y_{[0, t]}, \check{w}_{[0, t]}, \xi_{[0, t]}, \widehat{\xi}_{[0, t]}, \check{\xi}_{[0, t]}\right)\right|^{2} \\
& -\gamma^{2}\left|v-\bar{v}_{2}\right|^{2} .
\end{aligned}
$$

Then the optimal choice for the variables $\xi_{c}$ and $\widehat{\xi}$ are

$$
\xi_{c}^{*}=-\frac{1}{2} \bar{\varsigma}_{2} \Longleftrightarrow \widehat{\xi}^{*}=\check{\xi}-\frac{1}{2} \bar{\varsigma}_{2}
$$

which yields that the closed-loop system is dissipative with storage function $U$ and supply rate

$$
-\left|x_{1}-y_{d}\right|^{2}+\gamma^{2}|w|^{2}+\gamma^{2}|\check{w}|^{2}
$$

Furthermore, the worst case disturbance with respect to the value function $U$ is given by

$$
\begin{aligned}
w_{\mathrm{opt}}= & \zeta E^{\prime} e_{2,1}^{\prime} \bar{v}_{2}+\frac{1}{\gamma^{2}}\left(I-\zeta^{2} E^{\prime} E\right) \bar{D}^{\prime} \bar{\Sigma}^{-1}(\xi-\check{\xi}) \\
& +\zeta^{2} E^{\prime} C(\check{x}-x), \\
\check{w}_{\mathrm{opt}}= & e_{2,2} \bar{v}_{2} .
\end{aligned}
$$

\section{Main Result}

For the adaptive control law with $\xi_{c}$ chosen according to (65), the closed-loop system dynamics are

$$
\dot{X}=F\left(X, y_{d}^{(2)}\right)+G(X) w+G_{\check{w}}(X) \check{w} .
$$

$X$ is the state vector of the close-loop system and given by

$$
X:=\left[\begin{array}{lllllllllllll}
\theta^{\prime} & x^{\prime} & s_{\Sigma} & \check{\theta}^{\prime} & \check{x}^{\prime} & \eta^{\prime} & \eta_{d}^{\prime} & \eta_{\check{w}}^{\prime} & \vec{\Phi}_{u}^{\prime} & \dot{\Sigma}^{\prime} & y_{d} & y_{d}^{(1)}
\end{array}\right]^{\prime},
$$

which belongs to the set $\mathscr{D}:=\left\{X \mid \Sigma>0, s_{\Sigma}>0, \check{\theta} \in \Theta_{o}\right\} ; F$ and $G$ are smooth mapping of $\mathscr{D} \times \mathbb{R}$ and $\mathscr{D}$, respectively, and with the initial condition $X(0)=X_{0} \in \mathscr{D}_{0}:=\left\{X_{0} \in \mathscr{D} \mid \theta \in\right.$ $\Theta, \check{\theta}_{0} \in \Theta, \Sigma(0)=\gamma^{-2} Q_{0}^{-1}>0, \operatorname{Tr}\left((\Sigma(0))^{-1}\right) \leq K_{c}, s_{\Sigma}(0)=$ $\left.\gamma^{2} \operatorname{Tr}\left(Q_{0}\right)\right\}$.

Since (64) holds, the value function $U$ satisfies HamiltonJacobi-Isaacs equation, for all $X \in \mathscr{D}$, for all $y_{d}^{(2)} \in \mathbb{R}$.

$$
\begin{aligned}
\frac{\partial U}{\partial X}(X) F\left(X, y_{d}^{(2)}\right)+\frac{1}{4 \gamma^{2}} \frac{\partial U}{\partial X}(X)\left[G(X) G_{w}(X)\right] \\
\cdot\left[G(X) G_{w}(X)\right]^{\prime}\left(\frac{\partial U}{\partial X}(X)\right)^{\prime} \\
+Q\left(X, y_{d}^{(2)}\right)=0
\end{aligned}
$$


where $Q: \mathscr{D} \times \mathbb{R} \rightarrow \mathbb{R}$ is smooth and given by

$$
\begin{array}{r}
Q\left(X, y_{d}^{(2)}\right)=\left|x_{1,1}-y_{d}\right|^{2}+\left(|\xi-\check{\xi}|_{\overline{\mathrm{Q}}}^{2}+P_{r}(\check{\theta})|\widehat{\eta}|_{Y}^{2}-2(\theta-\check{\theta})^{\prime}\right. \\
\left.\times P_{r}(\check{\theta})+\sum_{j=1}^{2} \beta_{j} z_{j}^{2}+\frac{1}{4}\left|\varsigma_{2}\right| \overline{\mathrm{Q}}\right) .
\end{array}
$$

Although the value function $U$ satisfies an HamiltonJacobi-Isaacs equation, we cannot deduce the stability and robustness properties of the closed-loop system directly from (64), since $U$ is not a positive-definite function of the closedloop state vector $X$. We will use the following theorem to precisely state the strong stability properties of the closedloop adaptive system.

Theorem 10. Consider the robust adaptive control problem formulated in Section 2 with Assumptions 1-7 holding. The robust adaptive controller $\mu$ defined by (60) with the optimal choice for the worst-case estimate $\widehat{\xi}$ defined by (65) achieves the following strong robustness properties for the closed-loop system:

(1) The controller $\mu$ achieves disturbance attenuation level $\gamma$ for any uncertainty quadruple $\left(x(0), \theta, w_{[0, \infty)}\right.$, $\left.\check{w}_{[0, \infty)}, Y_{d 0}, y_{d}^{(2)}\right) \in \mathscr{W}$.

(2) Given a $c_{w}>0$, there exists a constant $c_{c}>0$ and $a$ compact set $\Theta_{c} \subset \Theta_{o}$, such that for any uncertainty $\left(x(0), \theta, \grave{w}_{[0, \infty)}, \check{w}_{[0, \infty)}, Y_{d}\right)$ with

$$
\begin{gathered}
|x(0)| \leq c_{w} ; \quad|\grave{w}(t)| \leq c_{w} ; \quad|\check{w}(t)| \leq c_{w} ; \\
\left|Y_{d}(t)\right| \leq c_{w} ; \quad \forall t \in[0, \infty)
\end{gathered}
$$

all closed-loop state variables $x, \check{x}, \check{\theta}, \Sigma, s_{\Sigma}, \eta, \eta^{\check{w}}, \eta_{d}$, and $\lambda$ are bounded as follows, for all $t \in[0, \infty)$,

$$
\begin{gathered}
|x(t)| \leq c_{c}, \quad|\check{x}(t)| \leq c_{c}, \quad \check{\theta}(t) \in \Theta_{c}, \quad|\eta(t)| \leq c_{c}, \\
\left|\eta_{d}(t)\right| \leq c_{c}, \quad|\lambda(t)| \leq c_{c}, \quad\left|\eta^{\check{w}}\right| \leq c_{c}, \\
\frac{1}{K_{c}} I \leq \Sigma(t) \leq \frac{1}{\gamma^{2}} Q_{0}^{-1}, \quad \frac{1}{K_{c}} \leq s_{\Sigma}(t) \leq \frac{1}{\gamma^{2} \operatorname{Tr}\left(Q_{0}\right)} .
\end{gathered}
$$

(3) For any uncertainty quadruple $\left(x(0), \theta, \grave{w}_{[0, \infty)}, \check{w}_{[0, \infty)}\right.$, $\left.Y_{d[0, \infty)}\right)$ with $\grave{w}_{[0, \infty)} \in \mathscr{L}_{2} \cap \mathscr{L}_{\infty}, \check{w}_{[0, \infty)} \in \mathscr{L}_{2} \cap \mathscr{L}_{\infty}$ and $Y_{d[0, \infty)} \in \mathscr{L}_{\infty}$, the output of the system, $x_{1}$, asymptoti-cally tracks the reference trajectory, $y_{d}$, that is,

$$
\lim _{t \rightarrow \infty}\left(x_{1}(t)-y_{d}(t)\right)=0
$$

Proof. For the frits statement, if we define

$$
\begin{aligned}
l_{0}\left(\check{x}_{0}, \check{\theta}_{0}\right):= & \bar{V}_{2}(0)=\frac{1}{2} \sum_{j=1}^{2} z_{j}^{2}(0) \\
l\left(\tau, \theta, x, y_{[0, t]}, \check{w}_{[0, t]}\right):= & |\xi-\widehat{\xi}|_{\overline{\widetilde{Q}}}^{2}-2(\theta-\check{\theta})^{\prime} P_{r}(\check{\theta}) \\
& +\frac{1}{4}\left|\bar{\varsigma}_{2}\right|_{\overline{\mathrm{Q}}}^{2}+|\widetilde{\eta}|_{Y}^{2}+\sum_{j=1}^{2} \bar{\beta}_{j} z_{j}^{2} \\
= & \gamma^{4}|(x-\widehat{x})-\Phi(\theta-\widehat{\theta})|_{\Pi^{-1} \Delta \Pi^{-1}}^{2} \\
& +\epsilon\left(\gamma^{2} \zeta^{2}-1\right)|\theta-\widehat{\theta}|_{\Phi^{\prime} C^{\prime} C \Phi}^{2} \\
& -2(\theta-\check{\theta})^{\prime} P_{r}(\check{\theta})+\frac{1}{4}\left|\bar{\varsigma}_{2}\right|_{\overline{\widetilde{Q}}}^{2} \\
& +|\widetilde{\eta}|_{Y}^{2}+\sum_{j=1}^{2} \bar{\beta}_{j} z_{j}^{2},
\end{aligned}
$$

then, we have

$$
\begin{aligned}
J^{t} & =J^{t}+\int_{0}^{t} \dot{U} d \tau-U(t)+U(0) \\
& \leq-U(t) \leq 0
\end{aligned}
$$

It follows that

$$
\sup _{\left(x(0), \theta, w_{[0, \infty)}, \check{w}_{[0, \infty)}\right) \in \mathscr{W}} J^{t} \leq 0 .
$$

This establishes the first statement.

Next, we will prove the second statement. Define $\left[0, t_{f}\right)$ to be the maximal interval on which the closed-loop system admits a solution. We will show that $t_{f}$ is always $\infty$.

Fix $c_{w} \geq 0$, and $c_{d} \geq 0$, consider any uncertainty $\left(x_{0}, \theta, \grave{w}_{[0, \infty)}, \check{w}_{[0, \infty)}, Y_{d}(t)\right)$ that satisfies:

$$
\begin{gathered}
\left|x_{0}\right| \leq c_{w} ; \quad|\grave{w}(t)| \leq c_{w} ; \quad|\check{w}(t)| \leq c_{w} ; \\
\left|Y_{d}(t)\right| \leq c_{d} \quad \forall t \in[0, \infty) .
\end{gathered}
$$

We define $\left[0, T_{f}\right)$ to be the maximal length interval on which for the closed system there exists a solution that lies in its definition. Furthemore, from the estiamtion design step, $\Sigma$ and $s_{\Sigma}$ are uniformly upper bounded and uniformly bounded away from 0 as desired.

Introduce the vector of variables

$$
X_{e}:=\left[\begin{array}{lllll}
\check{\theta}^{\prime} & (\tilde{x}-\Phi \tilde{\theta})^{\prime} & \tilde{\eta}^{\prime} & z_{1} & z_{2}
\end{array}\right]^{\prime}
$$

and two nonnegtive and continuous functions defined on $\mathbb{R}^{6+\sigma}$

$$
\begin{aligned}
& U_{M}\left(X_{e}\right):=K_{c}|\widetilde{\theta}|^{2}+\gamma^{2}|\tilde{x}-\Phi \widetilde{\theta}|_{\Pi^{-1}}^{2}+|\widetilde{\eta}|_{Z}^{2}+\sum_{j=1}^{2} \frac{1}{2} z_{j}^{2}, \\
& U_{m}\left(X_{e}\right):=\gamma^{2}|\widetilde{\theta}|_{Q_{0}}^{2}+\gamma^{2}|\tilde{x}-\Phi \widetilde{\theta}|_{\Pi^{-1}}^{2}+|\widetilde{\eta}|_{Z}^{2}+\sum_{j=1}^{2} \frac{1}{2} z_{j}^{2},
\end{aligned}
$$


then, we have

$$
U_{m}\left(X_{e}\right) \leq U\left(t, X_{e}\right) \leq U_{M}\left(X_{e}\right), \quad \forall\left(t, X_{e}\right) \in\left[0, T_{f}\right) \times \mathbb{R}^{6+\sigma} .
$$

Since $U_{m}\left(X_{e}\right)$ is continuous, nonnegative definite and radially unbounded, then for all $\alpha \in \mathbb{R}$, the set $S_{1 \alpha}:=\left\{X_{e} \in \mathbb{R}^{6+\sigma} \mid\right.$ $\left.U_{m}\left(X_{e}\right) \leq \alpha\right\}$ is compact or empty. Since $|\grave{w}(t)| \leq c_{w}$, and $|\breve{w}(t)| \leq c_{w}$, for all $t \in[0, \infty)$, we have the following inequality for the derivative of $U$ :

$$
\begin{aligned}
\dot{U} \leq & -\frac{\gamma^{4}}{2}|x-\check{x}-\Phi(\theta-\check{\theta})|_{\Pi^{-1} \Delta \Pi^{-1}}^{2}+2(\theta-\check{\theta})^{\prime} P_{r}(\check{\theta}) \\
& -|\widetilde{\eta}|_{Y}^{2}-\sum_{i=1}^{2} \bar{\beta}_{i} z_{i}^{2}+\gamma^{2}\|\grave{M}\|_{2}^{2} c_{w}^{2}+\gamma^{2} c_{w}^{2} .
\end{aligned}
$$

Since $-\left(\gamma^{4} / 2\right)|x-\check{x}-\Phi(\theta-\check{\theta})|_{\Pi^{-1} \Delta \Pi^{-1}}^{2}-|\widetilde{\eta}|_{Y}^{2}+2(\theta-\check{\theta})^{\prime} P_{r}(\check{\theta})-$ $\sum_{j=1}^{2} \bar{\beta}_{j} z_{j}^{2}$ will tend to $-\infty$ when $X_{e}$ approaches the boundary of $\Theta_{o} \times \mathbb{R}^{6}$, then there exists a compact set $\Omega_{1}\left(c_{w}\right) \subset \Theta_{o} \times \mathbb{R}^{6}$, such that $\dot{U}<0$ for for all $X_{e} \in \Theta_{o} \times \mathbb{R}^{6} \backslash \Omega_{1}$. Then, $U\left(t, X_{e}(t)\right) \leq c_{1}$, and $X_{e}(t)$ is in the compact set $S_{1 c_{1}} \subseteq \mathbb{R}^{6+\sigma}$, for all $t \in\left[0, T_{f}\right)$. It follows that the signal $X_{e}$ is uniformly bounded, namely, $\widetilde{\theta}, \tilde{x}-\Phi \tilde{\theta}, \tilde{\eta}, z_{1}$, and $z_{2}$ are uniformly bounded.

Based on the dynamics of $\eta_{d}$, we have $\eta_{d}$ is uniformly bounded. Since $\tilde{\eta}=\eta-\eta_{d}$ is uniformly bounded, then $\eta$ is also uniformly bounded. Furthermore, there is a particular linear combination of the components of $\eta$, denoted by $\eta_{L}$,

$$
\begin{gathered}
\dot{\eta}=A_{f} \eta+p_{2} y, \\
\eta_{L}=T_{L} \eta,
\end{gathered}
$$

which is strictly minimum phase and has relative degree 1 with respect to $y$. Then the signal $\eta_{L}$ has relative degree 3 with respect to the input $u$, and is uniformly bounded.

Note $\Phi=\Phi^{y}+\Phi^{u}+\Phi^{\check{w}}$. Since $\Phi^{y}$ and $\Phi^{\check{w}}$ are uniformly bounded, to prove $\Phi$ is bounded, we need to prove $\Phi^{u}$ is uniformly bounded. Define the following equations to separate $\Phi^{u}$ into two parts:

$$
\begin{aligned}
\Phi^{u} & =\Phi_{u_{s}}+\lambda_{b} \bar{A}_{220}, \\
\lambda_{b} & =\left[\begin{array}{l}
\lambda_{b 1} \\
\lambda_{b 2}
\end{array}\right], \\
\dot{\lambda}_{b} & =A_{f} \lambda_{b}+e_{2,2} u ; \quad \lambda_{b}(0)=\mathbf{0}_{2 \times 1}, \\
\Phi_{u_{s}} & =\left[\begin{array}{l}
\Phi_{u_{s} 1} \\
\Phi_{u_{s}}
\end{array}\right], \\
\dot{\Phi}_{u_{s}} & =A_{f} \Phi_{u_{s}} ; \quad \Phi_{u_{s}}(0)=\Phi_{u 0} .
\end{aligned}
$$

Clearly $\Phi_{u_{s}}$ is uniformly bounded because $A_{f}$ is Hurwitz. The first-row element of $\tilde{x}-\Phi \widetilde{\theta}$ is

$$
\widetilde{x}_{1}-\Phi_{u_{s} 1} \tilde{\theta}-\lambda_{b 1} \bar{A}_{220} \tilde{\theta}-\Phi_{1}^{\check{\omega}} \tilde{\theta}-\eta^{\prime T_{1} \tilde{\theta}} .
$$

We can conclude that $\tilde{x}_{1}-\lambda_{b 1} \bar{A}_{220} \tilde{\theta}$ is uniformly bounded in view of the boundedness of $\tilde{x}-\Phi \widetilde{\theta}, \widetilde{\theta}, \Phi_{u_{s}}, \Phi^{\check{w}}$, and $\eta$. Since $z_{1}=\check{x}_{1}-y_{d}$, and $z_{1}, y_{d}$ are both uniformly bounded, $\check{x}_{1}$ is also uniformly bounded.

Notice that $A_{f}=A-\zeta^{2} L C-\Pi C^{\prime} C\left(\zeta^{2}-\gamma^{-2}\right)$ and $b_{0}=$ $b_{p 0}+\bar{A}_{220} \theta$, we generated the signal $x_{1}-b_{0} \lambda_{b 1}$ by

$$
\begin{aligned}
\dot{x}-b_{0} \dot{\lambda}_{b}= & A_{f}\left(x-b_{0} \lambda_{b}\right)+\bar{A}_{21} \theta y+D \grave{M} \grave{w}+\check{w} \bar{A}_{23} \theta \\
& +\left(\zeta^{2} L+\Pi C^{\prime}\left(\zeta^{2}-\frac{1}{\gamma^{2}}\right)\right)(y-E \grave{M} \grave{w})+\check{D} \check{w}, \\
x_{1}-b_{0} \lambda_{b 1}= & C\left(x-b_{0} \lambda_{b}\right) .
\end{aligned}
$$

Since $x_{1}-b_{0} \lambda_{b 1}$ has relative degree at least 1 with respect to $y$, take $\eta_{L}$ and $y$ as output and input of the reference system, we conclude $x_{1}-b_{0} \lambda_{b 1}$ is uniformly bounded by bounding lemma. It follows that $\check{x}_{1}-\lambda_{b 1}\left(b_{p 0}+A_{2120} \check{\theta}\right)$ is also uniformly bounded. Since $\check{x}_{1}$ is uniformly bounded and $\check{\theta}$ is uniformly bounded away from 0 , we have $\lambda_{b 1}$ is uniformly bounded. That further implies that $\Phi_{1}$, that is, $C \Phi$, is uniformly bounded. Furthermore, since $x_{1}-b_{0} \lambda_{b 1}, \check{w}$ and $\grave{w}$ are bounded, we have that the signals of $x_{1}$ and $y$ are uniformly bounded. It further implies the uniform boundedness of $x-b_{0} \lambda_{b}$ since $A_{f}$ is a Hurwitz matrix. By a similar line of reasoning above, we have $x_{2}, \lambda_{b, 2}$ are uniformly bounded. Then we can conclude that $\Phi_{u s}$ and $\Phi$ are uniformly bounded.

Next, we need to prove the existence of a compact set $\Theta_{c} \subset$ $\Theta_{o}$ such that $\check{\theta}(t) \in \Theta_{c}$, for all $t \in\left[0, T_{f}\right)$. First introduce the function

$$
\Upsilon:=U+\left(\rho_{o}-P(\check{\theta})\right)^{-1} P(\check{\theta}) .
$$

We notice that, when $\check{\theta}$ approaches the boundary of $\Theta_{o}, P(\check{\theta})$ approaches $\rho_{o}$. Then $\Upsilon$ approaches $\infty$ as $X_{e}$ approaches the boundary of $\Theta_{o} \times \mathbb{R}^{6}$. We introduce two nonnegative and continuous functions defined on $\Theta_{o} \times \mathbb{R}^{4}$ :

$$
\begin{aligned}
& \Upsilon_{M}:=U_{M}\left(X_{e}\right)+\left(\rho_{o}-P(\check{\theta})\right)^{-1} P(\check{\theta}), \\
& \Upsilon_{m}:=U_{m}\left(X_{e}\right)+\left(\rho_{o}-P(\check{\theta})\right)^{-1} P(\check{\theta}) .
\end{aligned}
$$

Then, by the previous analysis, we have

$$
\begin{aligned}
& \Upsilon_{m}\left(X_{e}\right) \leq \Upsilon\left(t, X_{e}\right) \leq \Upsilon_{M}\left(X_{e}\right), \\
& \forall\left(t, X_{e}\right) \in\left[0, T_{f}\right) \times \Theta_{o} \times \mathbb{R}^{6} .
\end{aligned}
$$

Note that the set $S_{2 \alpha}:=\left\{X_{e} \in \Theta_{o} \times \mathbb{R}^{6} \mid \Upsilon_{m}\left(X_{e}\right) \leq \alpha\right\}$ is a compact set or empty. Then, we consider the derivative 
of $\Upsilon$ as follows:

$$
\begin{aligned}
\dot{\Upsilon}= & \dot{U}+\left(\rho_{o}-P(\check{\theta})\right)^{-2} \rho_{o} \frac{\partial P}{\partial \theta}(\check{\theta}) \check{\theta} \\
\leq & -\frac{\gamma^{4}}{2}|x-\check{x}-\Phi(\theta-\check{\theta})|_{\Pi^{-1} \Delta \Pi^{-1}}^{2} \\
& +2(\theta-\check{\theta})^{\prime} P_{r}(\check{\theta})-|\widetilde{\eta}|_{Y}^{2}-\sum_{j=1}^{r} c_{\beta_{j}} z_{j}^{2} \\
& -\left|\left(\frac{\partial P}{\partial \theta}(\check{\theta})\right)^{\prime}\right|^{2}\left(\rho_{o}-P(\check{\theta})\right)^{-4} \\
& \times\left(K_{c}^{-1} \rho_{o} p_{r}(\check{\theta})\left(\rho_{o}-P(\check{\theta})\right)^{2}-c\right)+c,
\end{aligned}
$$

where $c \in \mathbb{R}$ is a positive constant. Since $\grave{\Upsilon}$ will tend to $-\infty$ when $X_{e}$ approaches the boundary of $\Theta_{o} \times \mathbb{R}^{4}$, there exists a compact set $\Omega_{2}\left(c_{w}\right) \subset \Theta_{o} \times \mathbb{R}^{4}$, such that for all $X_{e} \in \Theta_{o} \times \mathbb{R}^{4} \backslash$ $\Omega_{2}, \dot{\Upsilon}\left(X_{e}\right)<0$. Then, there exists a compact set $\Theta_{c} \subset \Theta_{o}$, such that $\check{\theta}(t) \in \Theta_{c}$, for all $t \in\left[0, T_{f}\right)$. Moreover, $\Upsilon\left(t, X_{e}(t)\right) \leq c_{2}$, and $X_{e}(t)$ is in the compact set $S_{2 c_{2}} \subseteq \Theta_{o} \times \mathbb{R}^{6}$, for all $t \in$ $\left[0, T_{f}\right)$. It follows that $P_{r}(\check{\theta})$ is also uniformly bounded.

Also, $\eta, \lambda$ are some stably filtered signals of $u$ and $y$, they are uniformly bounded. Since $\eta^{\check{w}}$ is uniformly bounded, $\Phi$ is uniformly bounded. Then we can conclude $\check{x}$ is uniformly bounded from the boundedness of $\tilde{x}-\Phi \widetilde{\theta}$. This further implies that the control input $u$ is uniformly bounded.

Then we can get the conclusion that the complete system states and $u$ are uniformly bounded on $\left[0, t_{f}\right), \Sigma, s_{\Sigma}$ are uniformly bounded and bounded away from 0 , and $\check{\theta}$ is uniformly bounded away from the boundary of the set $\Theta_{o}$. Therefore, it follows that $t_{f}=\infty$ and the complete system states are uniformly bounded on $[0, \infty)$.

Last, we will establish the third statement. By the following inequality:

$$
\int_{0}^{\infty} \dot{U} d \tau \leq \int_{0}^{\infty}\left(-\left|x_{1}-y_{d}\right|^{2}+\gamma^{2}|\grave{M} \grave{w}|^{2}+\gamma^{2}|\check{w}|^{2}\right) d \tau,
$$

it follows that

$$
\begin{aligned}
& \int_{0}^{\infty}\left|x_{1}-y_{d}\right|^{2} d \tau \\
& \quad \leq \int_{0}^{\infty}\left(\gamma^{2}|\grave{M} \grave{w}|^{2}+\gamma^{2}|\check{w}|^{2}\right) d \tau+U(0)<+\infty .
\end{aligned}
$$

By the second statement, we notice that

$$
\sup _{0 \leq t<\infty}\left|\dot{x}_{1}-\dot{y}_{d}\right|<\infty
$$

Then we have

$$
\lim _{t \rightarrow \infty}\left|x_{1}(t)-y_{d}(t)\right|=0
$$

This complete the proof of the theorem.

\section{Example}

In this section, we present one example to illustrate the main results of this paper. The designs were carried out using MATLAB symbolic computation tools, and the closed-loop systems were simulated using SIMULINK.

The example was based on a four-pole-permanentmagnet brushed DC motor. We assume that the nominal values of $K_{t}, K_{e}, J, R$, and $L$ are given as below, and the variations can be lumped into the arbitrary disturbance $\grave{w}$.

$$
\begin{aligned}
& K_{t}=0.01 ; \mathrm{N}-\mathrm{cm} / \mathrm{Amp}, \\
& K_{e}=1 ; \mathrm{Volt} / \mathrm{rad} / \mathrm{s}, \\
& J=0.01 ; \mathrm{N}-\mathrm{cm} / \mathrm{rad} / \mathrm{s} 2, \\
& R=1 ; \mathrm{Ohm}, \\
& L=0.1 ; \mathrm{L} .
\end{aligned}
$$

The value of $D$ is unknown and with true value $0.01 \mathrm{~N}$ $\mathrm{cm} / \mathrm{rad} / \mathrm{s}$. Then the true system is of the following state-space representation

$$
\begin{aligned}
{\left[\begin{array}{c}
\dot{\omega} \\
\dot{i}
\end{array}\right]=} & {\left[\begin{array}{cc}
\theta & 1 \\
-10 & -10
\end{array}\right]\left[\begin{array}{c}
\omega \\
i
\end{array}\right]+\left[\begin{array}{c}
0 \\
10
\end{array}\right] u+\left[\begin{array}{l}
1 \\
0
\end{array}\right] T_{\breve{w}} } \\
& +\left[\begin{array}{lll}
1 & 0 & 1 \\
0 & 0 & 0
\end{array}\right]\left[\begin{array}{c}
T_{w} \\
w_{\omega} \\
T_{f}
\end{array}\right] ; \quad\left[\begin{array}{c}
\omega(0) \\
i(0)
\end{array}\right]=\left[\begin{array}{l}
0 \\
0
\end{array}\right], \\
y= & {\left[\begin{array}{ll}
1 & 0
\end{array}\right]\left[\begin{array}{c}
\omega \\
i
\end{array}\right]+\left[\begin{array}{lll}
0 & 1 & 0
\end{array}\right]\left[\begin{array}{c}
T_{w} \\
w_{\omega} \\
T_{f}
\end{array}\right], }
\end{aligned}
$$

where $\omega$ is the motor speed in $\mathrm{rad} / \mathrm{s}, i$ is the motor current in amp, $u$ is control input in volt, $y$ is the motor speed measurement in $\mathrm{rad} / \mathrm{s}, T_{\breve{w}}$ is the estimated disturbance torque in $\mathrm{N}-\mathrm{cm}, T_{w}$ is the arbitrary disturbance torque in $\mathrm{N}-\mathrm{cm}, T_{f}$ is the friction torque in $\mathrm{N}-\mathrm{cm}, w_{\omega}$ is the measurement channel noise in $\mathrm{rad} / \mathrm{s}, \theta$ is the 1-dimensional unknown parameter with the true value $\theta^{*}=-1$ belonging to the interval $[-2,0]$.

The control objective is to have the system output tracking velocity reference trajectory, $y_{d}$, which is generated by the following linear system:

$$
y_{d}=\frac{d}{s^{3}+2 s^{2}+2 s^{+} 3}
$$

where $d$ is the command input signal.

Introduce the following state and disturbance transformation:

$$
x=\left[\begin{array}{cc}
1 & 0 \\
10 & 1
\end{array}\right]\left[\begin{array}{c}
\omega \\
i
\end{array}\right] ; \quad w=\left[\begin{array}{ccc}
1 & -\theta & 1 \\
0 & 1 & 0
\end{array}\right]\left[\begin{array}{c}
T_{w} \\
w_{\omega} \\
T_{f}
\end{array}\right] .
$$

We obtain the design model

$$
\begin{aligned}
\dot{x}= & {\left[\begin{array}{ll}
-10 & 1 \\
-10 & 0
\end{array}\right] x+\left[\begin{array}{c}
1 \\
10
\end{array}\right] y \theta } \\
& +\left[\begin{array}{c}
0 \\
10
\end{array}\right] u+\left[\begin{array}{c}
1 \\
10
\end{array}\right] \check{w}+\left[\begin{array}{cc}
1 & 0 \\
10 & 0
\end{array}\right] w, \\
y= & {\left[\begin{array}{ll}
1 & 0
\end{array}\right] x+\left[\begin{array}{ll}
0 & 1
\end{array}\right] w . }
\end{aligned}
$$




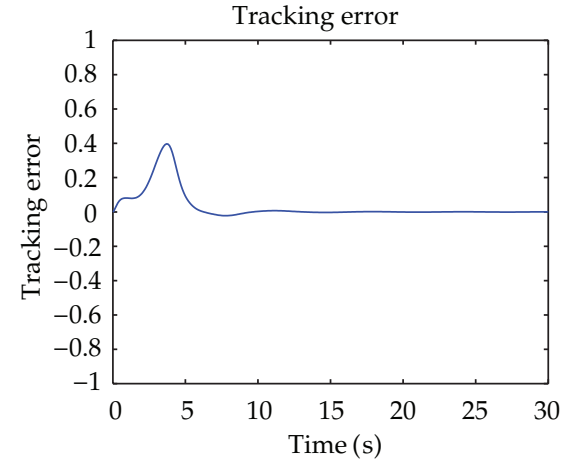

(a)

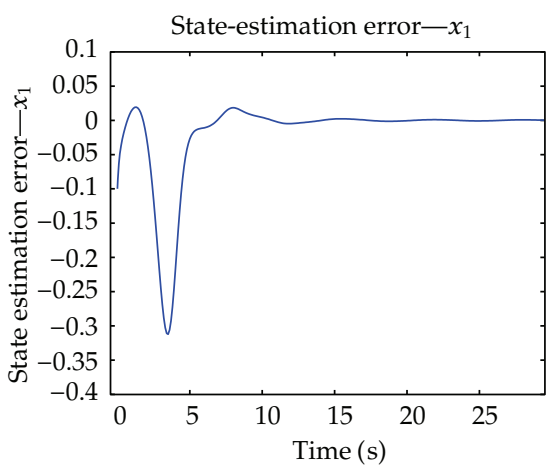

(d)

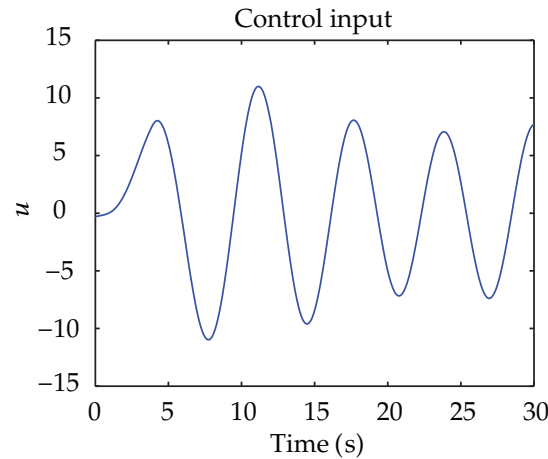

(b)

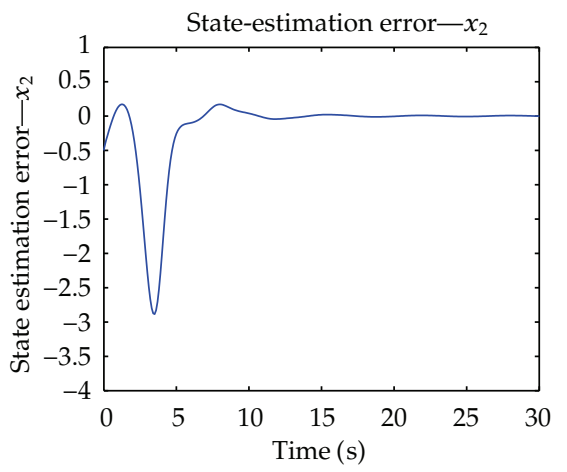

(e)

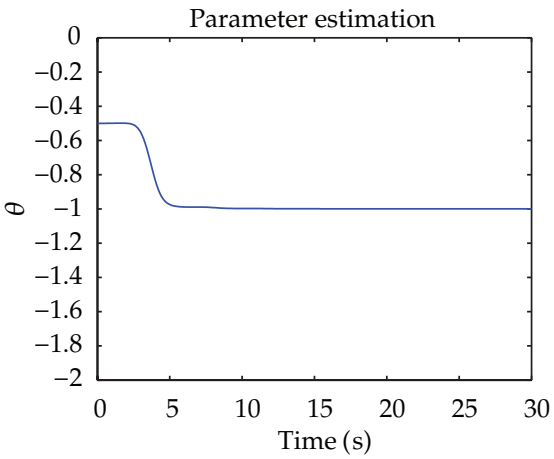

(c)

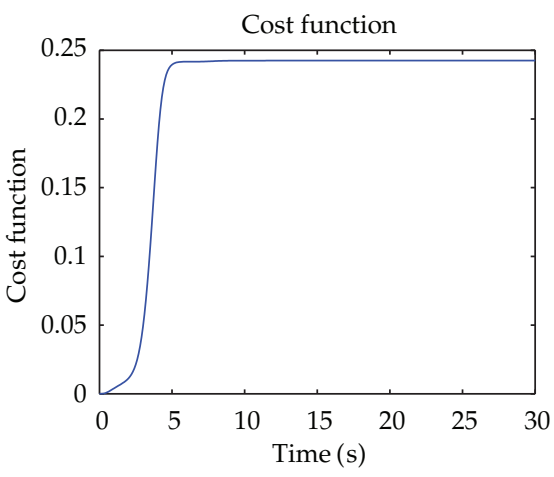

(f)

FIGURE 1: System response for example under command input $d(t)=5 \sin (t), T_{f}=0, T_{w}=0, w_{\omega}=0$, and $T_{\check{w}}=0$. (a) Tracking error; (b) control input; (c) parameter estimate; (d) state-estimation error $\check{x}_{1}-x_{1}$; (e) state-estimation error $\check{x}_{2}-x_{2} ;(\mathrm{f})$ cost function $\int_{0}^{t}\left(\left(x_{1}-y_{d}\right)^{2}-\right.$ $\left.\gamma^{2}|w|^{2}-\gamma^{2}|\check{w}|^{2} \mathrm{~d}\right) \tau$.

The ultimate performance lower bound for this system is 1 with respect to $w$. For the adaptive control design, we set the desired disturbance attenuation level $\gamma=\sqrt{2}$. The parameter $\theta$ is assumed to belong to the set $[-2,0]$, with the projection function $P(\theta)$ chosen as

$$
P(\theta)=(\theta+1)^{2} .
$$

For other design and simulation parameters, we select

$$
\begin{gathered}
\check{x}_{0}=\left[\begin{array}{l}
0.1 \\
0.5
\end{array}\right] ; \quad \check{\theta}_{0}=-0.5 ; \\
Q_{0}=1 ; \quad K_{c}=100 ; \quad \Delta=\left[\begin{array}{ll}
1 & 0 \\
0 & 1
\end{array}\right] ; \\
\bar{\beta}_{1}=\bar{\beta}_{2}=0.5 ; \quad Y=\left[\begin{array}{cc}
159.2262 & -17.0150 \\
-17.0150 & 1.8786
\end{array}\right] .
\end{gathered}
$$

Then, we obtain

$$
\begin{gathered}
A_{f}=\left[\begin{array}{cc}
-10.2993 & 1.0000 \\
-12.2882 & 0
\end{array}\right] ; \quad Z=\left[\begin{array}{cc}
8.8506 & -0.9393 \\
-0.9393 & 0.1229
\end{array}\right] ; \\
\Pi=\left[\begin{array}{cc}
0.5987 & 4.5764 \\
4.5764 & 43.1208
\end{array}\right] .
\end{gathered}
$$

We present two sets of simulation results in this example. In the first set of simulation, we set

$$
\begin{aligned}
& T_{f}=0 ; \mathrm{N}-\mathrm{cm}, \\
& T_{w}=0 ; \mathrm{N}-\mathrm{cm}, \\
& w_{\omega}=0 ; \mathrm{rad} / \mathrm{s}, \\
& T_{\check{\omega}}=0 ; \mathrm{N}-\mathrm{cm} .
\end{aligned}
$$

This simulation is to demonstrate the regulatory behaviour of the adaptive controller. The results are shown in Figures 1(a)1(f). We observe from Figure 1 that the parameter estimate of $-D / J$ asymptotically converges to its true value -1 , the output-tracking error and state-estimation error asymptotically converge to zeros and $t$ within 20 second. The control input is bounded by 12 , and the transient of the system is well behaved.

The second set of simulation results is to demonstrate the robustness of the adaptive controller to unmodeled exogenous disturbance inputs. We set

$$
\begin{aligned}
& T_{f}=-0.01 \times \operatorname{sgn}(\omega) ; \mathrm{N}-\mathrm{cm}, \\
& T_{w}=0.04 \sin (t) ; \mathrm{N}-\mathrm{cm}, \\
& w_{\omega}=\text { White noise signal with power } 0.01 \text { sample } d \text { at } \\
& 1 \mathrm{HZ} ; \mathrm{rad} / \mathrm{s}, \\
& T_{\breve{w}}=0.05 \sin (4 t) ; \mathrm{N}-\mathrm{cm} .
\end{aligned}
$$




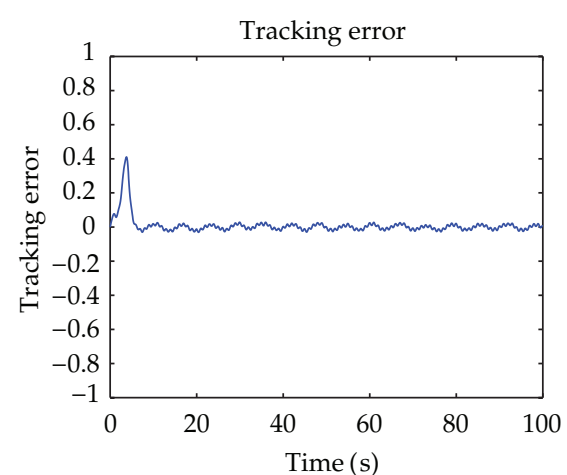

(a)

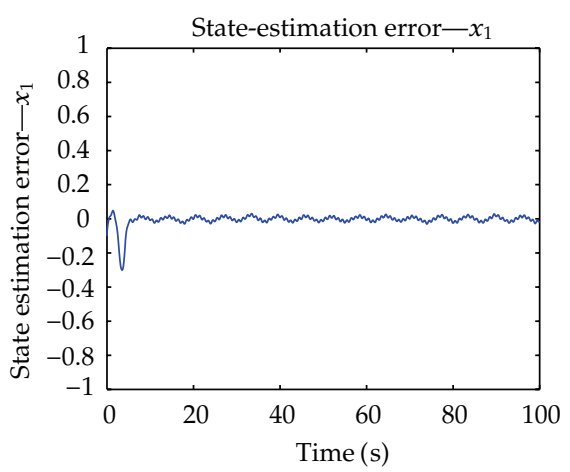

(d)

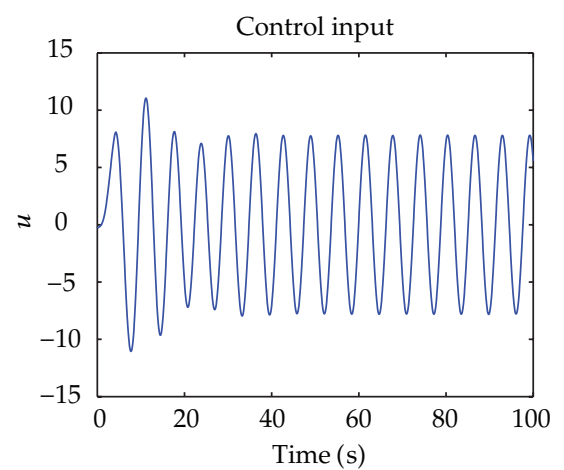

(b)

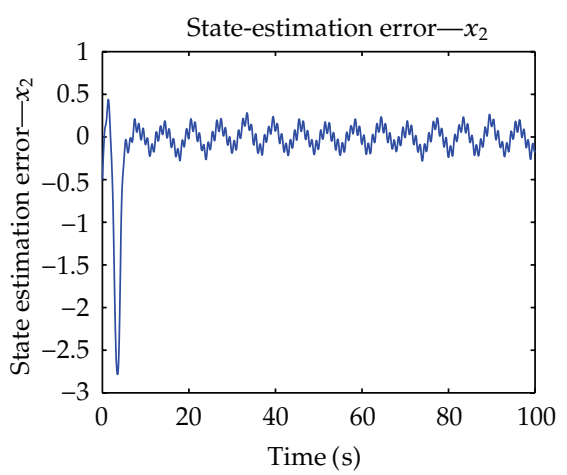

(e)

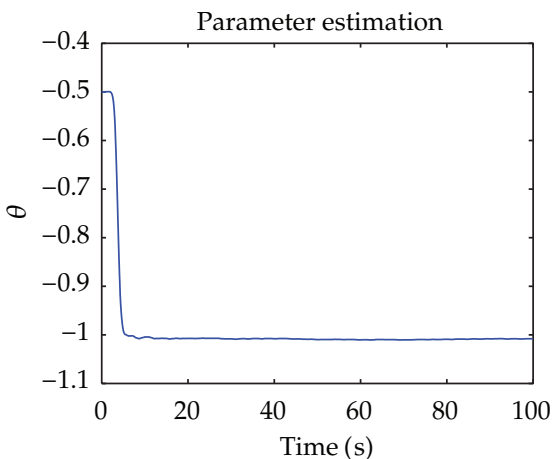

(c)

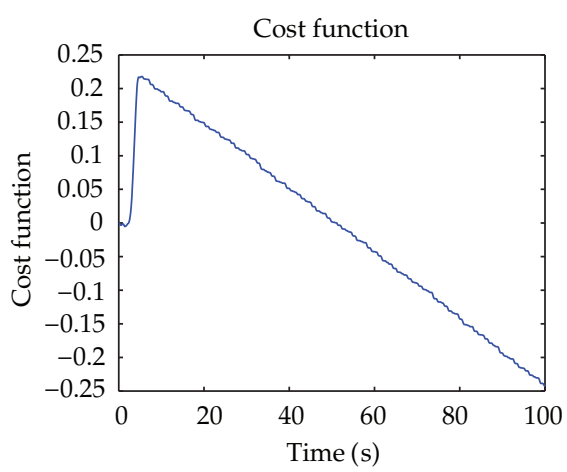

(f)

Figure 2: System response for example under command input $d(t)=5 \sin (t), T_{f}=-0.01 \times \operatorname{sgn}(\omega)$, and $T_{w}=0.04$ sin $(t)$, $w_{\omega}=$ white noise signal with power 0.01 sample $d$ at $1 \mathrm{HZ}, T_{\breve{w}}=0.05 \sin (4 t)$. (a) Tracking error; (b) control input; (c) parameter estimate; (d) state-estimation error $\check{x}_{1}-x_{1} ;(\mathrm{e})$ state-estimation error $\check{x}_{2}-x_{2} ;(\mathrm{f})$ cost function $\int_{0}^{t}\left(\left(x_{1}-y_{d}\right)^{2}-\gamma^{2}|w|^{2}-\gamma^{2}|\check{w}|^{2} \mathrm{~d}\right) \tau$.

The simulation results are presented in Figures 2(a)-2(f). We observe that the the parameter estimate of $-D / J$ no longer converges to the true value -1 , but it's stabilized around the true value. The output-tracking error and state-estimation error no longer converge to zeros, but output-tracking error satisfies the targeted attenuation level based on Figure 2(f), and the state-estimation errors asymptotically oscillate around zeros. The control input is again bounded by 12 , and the transient of the system is well behaved as well.

\section{Conclusions}

In this paper, we studied the permanent magnet brushed DC adaptive control design for velocity tracking applications. We formulate the robust adaptive control problem as a nonlinear $H^{\infty}$-control problem under imperfect state measurements, and then use cost-to-come function analysis and the integrator backstepping methodology to obtain the controller. The controller then achieves the desired disturbance attenuation level, with the ultimate lower bound of the attenuation level being the noise intensity in the measurement channel. It also guarantees the total stability of the closed-loop system and achieves asymptotic tracking of the reference trajectory when the disturbance is of finite energy and uniformly bounded.

\section{References}

[1] G. C. Goodwin and K. S. Sin, Adaptive Filtering, Prediction and Control, Prentice-Hall, Englewood Cliffs, NJ, USA, 1984.

[2] G. C. Goodwin and D. Q. Mayne, "A parameter estimation perspective of continuous time model reference adaptive control," Automatica, vol. 23, no. 1, pp. 57-70, 1987.

[3] P. R. Kumar, "A survey of some results in stochastic adaptive control," SIAM Journal on Control and Optimization, vol. 23, no. 3, pp. 329-380, 1985.

[4] C. E. Rohrs, L. Valavani, M. Athans, and G. Stein, "Robustness of continuous-time adaptive control algorithms in the presence of unmodeled dynamics," IEEE Transactions on Automatic Control, vol. 30, no. 9, pp. 881-889, 1985.

[5] A. Datta and P. A. Ioannou, "Performance analysis and improvement in model reference adaptive control," IEEE Transactions on Automatic Control, vol. 39, no. 12, pp. 2370-2387, 1994.

[6] P. A. Ioannou and J. Sun, Robust Adaptive Control, Prentice Hall, Upper Saddle River, NJ, USA, 1996.

[7] A. S. Morse, "Supervisory control of families of linear set-point controllers. I. Exact matching," IEEE Transactions on Automatic Control, vol. 41, no. 10, pp. 1413-1431, 1996.

[8] E. Mosca and T. Agnoloni, "Inference of candidate loop performance and data filtering for switching supervisory control," Automatica, vol. 37, no. 4, pp. 527-534, 2001. 
[9] A. Bilbao-Guillerna, M. De la Sen, A. Ibeas, and S. AlonsoQuesada, "Robustly stable multiestimation scheme for adaptive control and identification with model reduction issues," Discrete Dynamics in Nature and Society, no. 1, pp. 31-67, 2005.

[10] N. Luo, M. de la Sen, and J. Rodellar, "Robust stabilization of a class of uncertain time delay systems in sliding mode," International Journal of Robust and Nonlinear Control, vol. 7, no. 1, pp. 59-74, 1997.

[11] T. Başar and P. Bernhard, $H^{\infty}$-Optimal Control and Related Minimax Design Problems, Systems \& Control: Foundations \& Applications, Birkhäuser Boston Inc., Boston, MA, Second edition, 1995, A dynamic game approach.

[12] Z. Pan and T. Başar, "Parameter identification for uncertain linear systems with partial state measurements under an $H^{\infty}$ criterion," IEEE Transactions on Automatic Control, vol. 41, no. 9, pp. 1295-1311, 1996.

[13] I. E. Tezcan and T. Basar, "Disturbance attenuating adaptive controllers for parametric strict feedback nonlinear systems with output measurements," Journal of Dynamic Systems, Measurement and Control, Transactions of the ASME, vol. 121, no. 1, pp. 48-57, 1999.

[14] Z. . Pan and T. Basar, "Adaptive controller design and disturbance attenuation for SISO linear systems with noisy output measurements," CSL Report, University of Illinois at UrbanaChampaign, Urbana, Ill, USA, 2000.

[15] G. Arslan and T. Başar, "Disturbance attenuating controller design for strict-feedback systems with structurally unknown dynamics," Automatica, vol. 37, no. 8, pp. 1175-1188, 2001.

[16] S. Zeng and E. Fernandez, "Adaptive controller design and disturbance attenuation for sequentially interconnected SISO linear systems under noisy output measurements," IEEE Transactions on Automatic Control, vol. 55, no. 9, pp. 2123-2129, 2010.

[17] Q. Zhao, Z. Pan, and E. Fernandez, "Convergence analysis for reduced-order adaptive controller design of uncertain SISO linear systems with noisy output measurements," International Journal of Control, vol. 82, no. 11, pp. 1971-1990, 2009.

[18] Q. Zhao, Z. Pan, and E. Fernandez, "Reduced-order robust adaptive control design of uncertain SISO linear systems," International Journal of Adaptive Control and Signal Processing, vol. 22, no. 7, pp. 663-704, 2008.

[19] S. Zeng, "Adaptive controller design and disturbance attenuation for a general class of sequentially interconnected SISO linear systems with noisy output measurements," in Proceedings of the 49th IEEE Conference on Decision and Control (CDC '10), pp. 2608-2613, Atlanta, Ga, USA, December 2010.

[20] S. Zeng, "Adaptive controller design and disturbance attenuation for a general class of sequentially interconnected siso linear systems with noisy output measurements and partly measured disturbances," in Proceedings of the IEEE International Symposium on Computer-Aided Control System Design (CACSD) Part of 2011 IEEE Multi-Conference on Systems and Control, pp. 10501055, Denver, Colo, USA, 2011.

[21] S. Zeng, "Worst-case analysis based adaptive control design for siso linear systems with plant and actuation uncertainties," in Proceedings of the 50th IEEE Conference on Decision and Control and European Control Conference (CDC-ECC '11), pp. 63496354, Orlando, Fla, USA, 2011.

[22] S. Zeng and Z. Pan, "Adaptive controls design and disturbance attenuation for SISO linear systems with noisy output measurements and partly measured disturbances," International Journal of Control, vol. 82, no. 2, pp. 310-334, 2009.
[23] S. Zeng, Z. Pan, and E. Fernandez, "Adaptive controller design and disturbance attenuation for SISO linear systems with zero relative degree under noisy output measurements," International Journal of Adaptive Control and Signal Processing, vol. 24, no. 4, pp. 287-310, 2010.

[24] M. Krstić, I. Kanellakopoulos, and P. V. Kokotović, Nonlinear and Adaptive Control Design, John Wiley \& Sons, New York, NY, USA, 1995. 


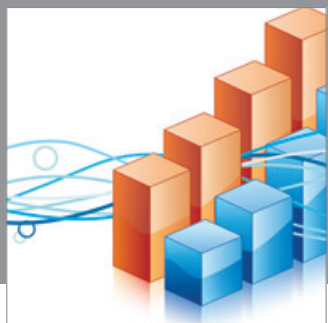

Advances in

Operations Research

mansans

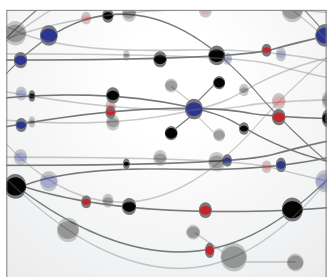

The Scientific World Journal
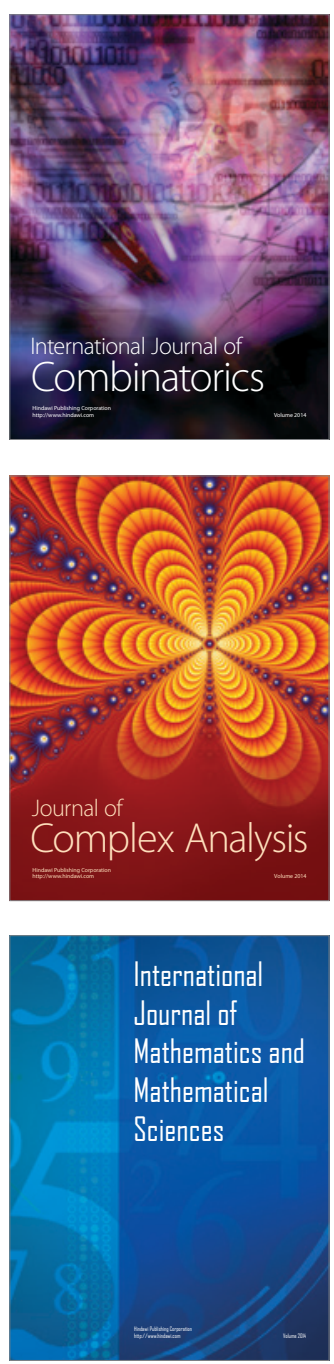
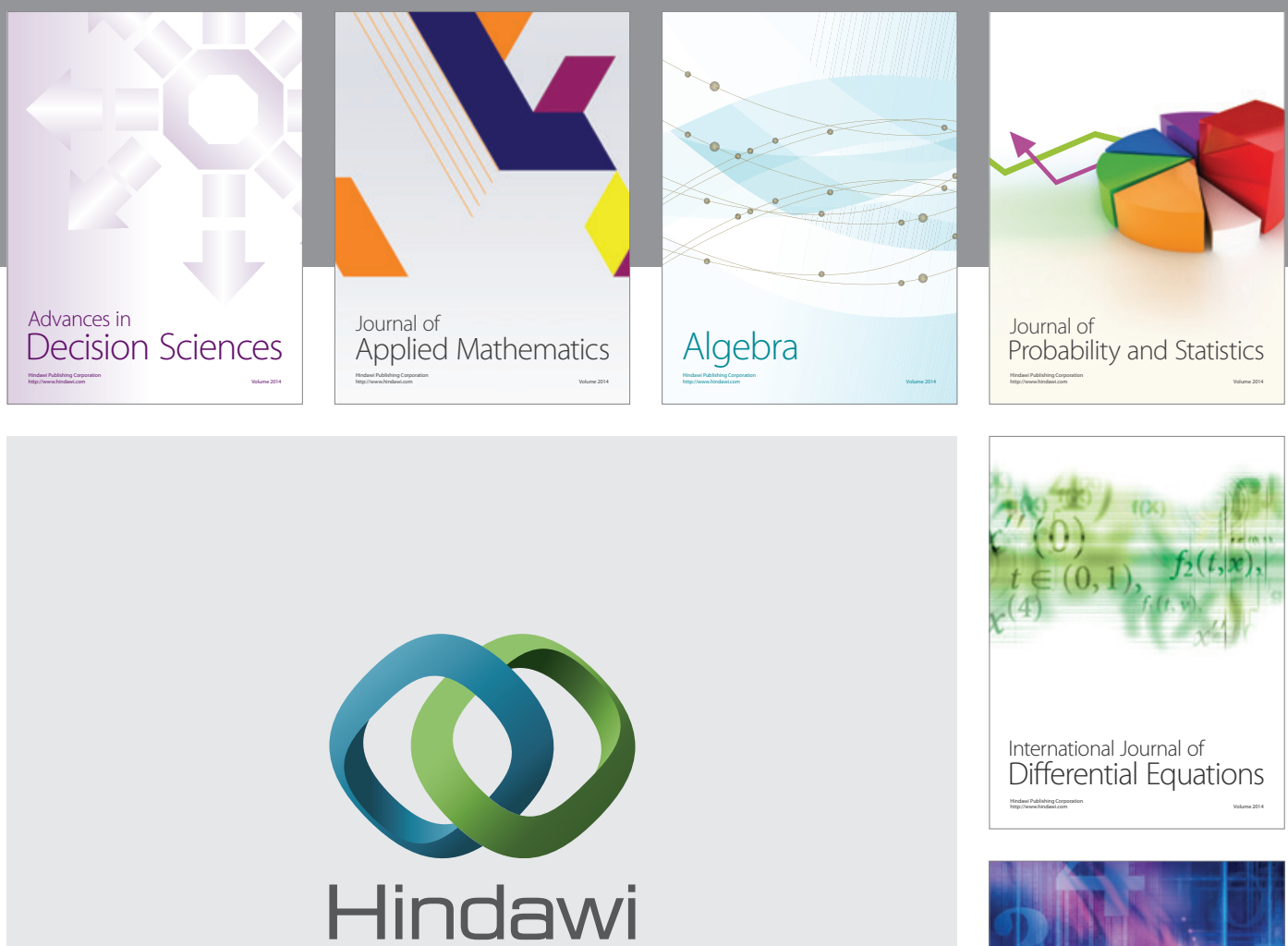

Submit your manuscripts at http://www.hindawi.com
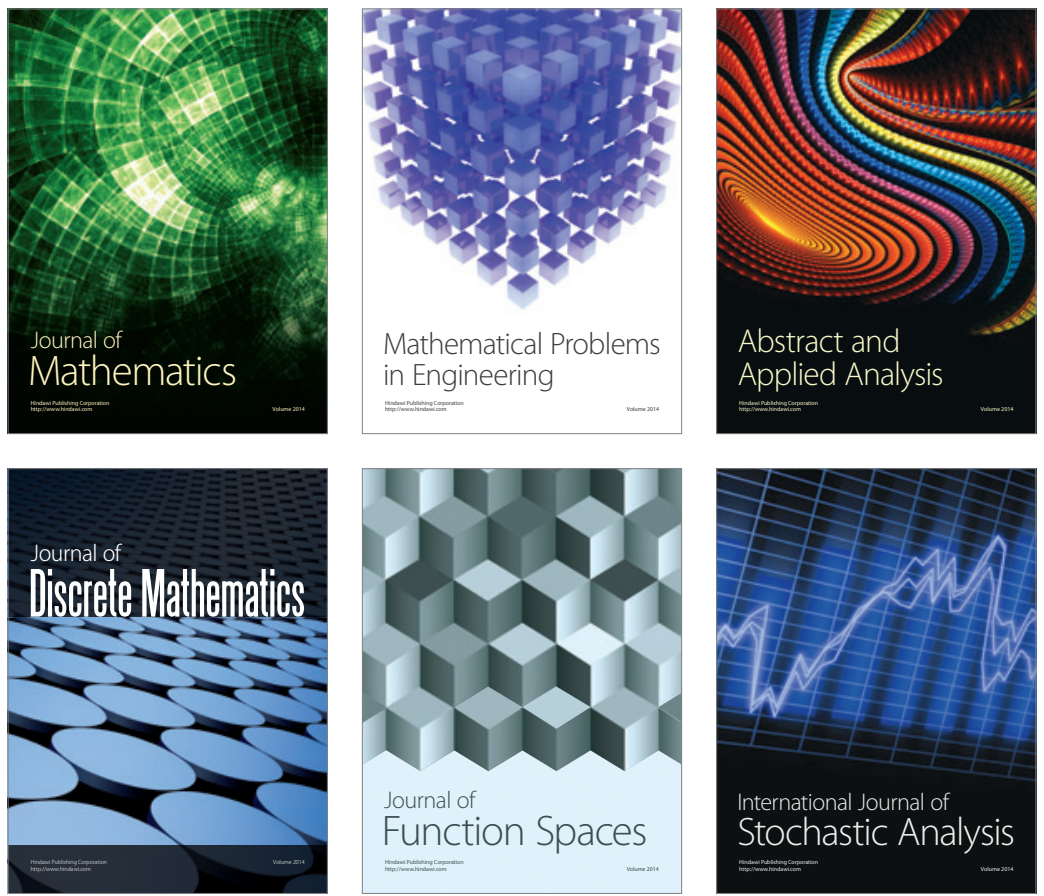

Journal of

Function Spaces

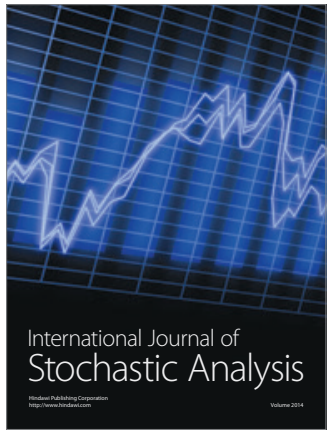

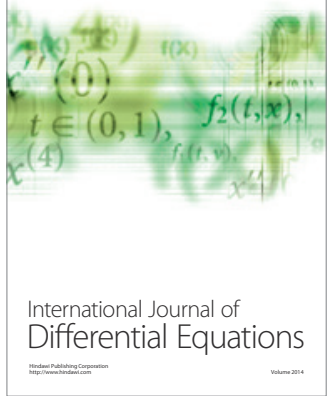
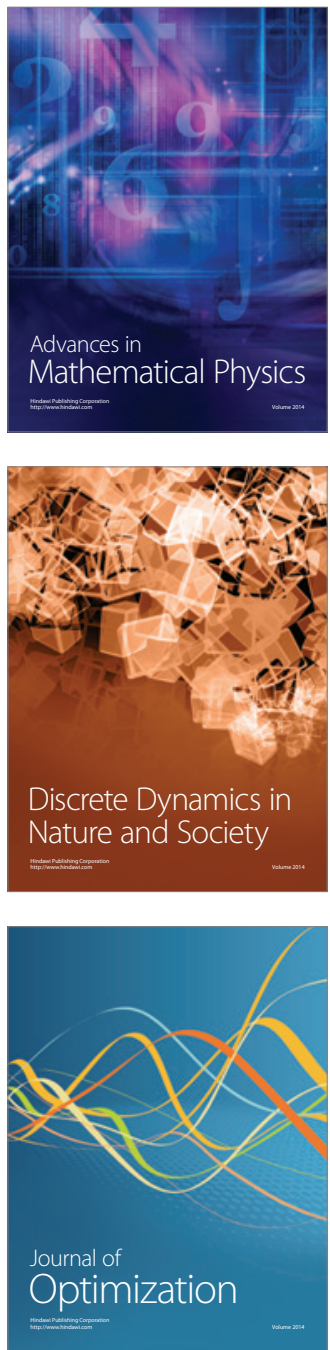